Article

\title{
Incidence and Impact of Acute Kidney Injury in Patients Receiving Extracorporeal Membrane Oxygenation: A Meta-Analysis
}

\author{
Charat Thongprayoon ${ }^{1}$, Wisit Cheungpasitporn ${ }^{2}{ }^{(\mathbb{D}}$, Ploypin Lertjitbanjong ${ }^{3}$, \\ Narothama Reddy Aeddula ${ }^{4}$ (D), Tarun Bathini ${ }^{5}$, Kanramon Watthanasuntorn ${ }^{3}$, Narat Srivali ${ }^{6}$, \\ Michael A. Mao ${ }^{7}$ (D) and Kianoush Kashani ${ }^{1,8, *}$ \\ 1 Division of Nephrology and Hypertension, Mayo Clinic, Rochester, MN 55905, USA \\ 2 Division of Nephrology, Department of Medicine, University of Mississippi Medical Center, Jackson, MS \\ 39216, USA \\ 3 Department of Internal Medicine, Bassett Medical Center, Cooperstown, NY 13326, USA \\ 4 Division of Nephrology, Department of Medicine, Deaconess Health System, Evansville, IN 47747, USA \\ Department of Internal Medicine, University of Arizona, Tucson, AZ 85721, USA \\ Division of Pulmonary and Critical Care Medicine, St. Agnes Hospital, Baltimore, MD 21229, USA \\ Division of Nephrology and Hypertension, Mayo Clinic, Jacksonville, FL 32224, USA \\ 8 Division of Pulmonary and Critical Care Medicine, Department of Medicine, Mayo Clinic, Rochester, MN \\ 55905, USA \\ * Correspondence: Kashani.Kianoush@mayo.edu; Tel.: +1-507-266-7093
}

Received: 15 May 2019; Accepted: 2 July 2019; Published: 5 July 2019

\begin{abstract}
Background: Although acute kidney injury (AKI) is a frequent complication in patients receiving extracorporeal membrane oxygenation (ECMO), the incidence and impact of AKI on mortality among patients on ECMO remain unclear. We conducted this systematic review to summarize the incidence and impact of AKI on mortality risk among adult patients on ECMO. Methods: A literature search was performed using EMBASE, Ovid MEDLINE, and Cochrane Databases from inception until March 2019 to identify studies assessing the incidence of AKI (using a standard AKI definition), severe AKI requiring renal replacement therapy (RRT), and the impact of AKI among adult patients on ECMO. Effect estimates from the individual studies were obtained and combined utilizing random-effects, generic inverse variance method of DerSimonian-Laird. The protocol for this systematic review is registered with PROSPERO (no. CRD42018103527). Results: 41 cohort studies with a total of 10,282 adult patients receiving ECMO were enrolled. Overall, the pooled estimated incidence of AKI and severe AKI requiring RRT were $62.8 \%$ (95\%CI: 52.1\%-72.4\%) and $44.9 \%$ (95\%CI: $40.8 \%-49.0 \%)$, respectively. Meta-regression showed that the year of study did not significantly affect the incidence of AKI $(p=0.67)$ or AKI requiring RRT ( $p=0.83)$. The pooled odds ratio (OR) of hospital mortality among patients receiving ECMO with AKI on RRT was 3.73 (95\% CI, 2.87-4.85). When the analysis was limited to studies with confounder-adjusted analysis, increased hospital mortality remained significant among patients receiving ECMO with AKI requiring RRT with pooled OR of 3.32 (95\% CI, 2.21-4.99). There was no publication bias as evaluated by the funnel plot and Egger's regression asymmetry test with $p=0.62$ and $p=0.17$ for the incidence of AKI and severe AKI requiring RRT, respectively. Conclusion: Among patients receiving ECMO, the incidence rates of AKI and severe AKI requiring RRT are high, which has not changed over time. Patients who develop AKI requiring RRT while on ECMO carry 3.7-fold higher hospital mortality.
\end{abstract}

Keywords: acute kidney injury; AKI; extracorporeal membrane oxygenation; ECMO; epidemiology; meta-analysis 


\section{Introduction}

Extracorporeal membrane oxygenation (ECMO), as a mechanical circulatory support system, is utilized as a treatment for cardiovascular or respiratory failure [1-3]. There are two main types of ECMO, including venovenous (VV)-ECMO for patients with isolated respiratory failure and venoarterial (VA)-ECMO for combined severe cardiac and respiratory failure [4]. Over the past 40 years, the clinical applications and feasibility of ECMO have expanded in patients with refractory cardiorespiratory failure, and there has been an exponential increase in the number of centers utilizing ECMO globally [3,5-9]. Studies have demonstrated survival benefits of ECMO ranging from $20 \%$ to $50 \%$ in patients with cardiac arrest, severe adult respiratory distress syndrome (ARDS), and refractory cardiogenic shock $[5,10-16]$.

Despite these benefits, there have been a number of reports to highlight the concomitant occurrence of organ failures and complications including acute kidney injury (AKI), infections, thrombosis, bleeding and coagulopathy, and neurological events $[17,18]$. The underlying mechanisms for AKI among patients requiring ECMO appear to be complex and include hemodynamic instabilities, inflammatory responses, coagulation-platelet abnormalities, and immune-mediated injury that arise from the primary underlying disease, premorbid conditions and the ECMO circuit [18-28]. Due to previously non-uniform definitions of AKI, the reported incidences of AKI among patients requiring ECMO therapy ranged widely from $8 \%$ up to $85 \%[4,7,15,18-70]$. In addition, the incidence and mortality associated with AKI in patients requiring ECMO and their trends remain unclear.

This systematic review was conducted with the aim to summarize the incidence (using standard AKI definitions) and the impact of AKI on mortality risk among adult patients on ECMO.

\section{Methods}

\subsection{Information Sources and Search Strategy}

The protocol for this systematic review and meta-analysis is registered with International Prospective Register of Systematic Reviews (PROSPERO no. CRD42018103527). A systematic literature review of EMBASE, Ovid MEDLINE, and the Cochrane Database of Systematic Reviews from database inception through March 2019 was conducted to summarize the incidence and impact of AKI on mortality risk among adult patients on ECMO. Two authors (C.T. and W.C.) independently performed a systematic literature search utilizing a search approach that consolidated the search terms "extracorporeal membrane oxygenation" OR "ECMO" AND "acute kidney injury" OR "acute renal failure." Further details regarding the search strategy utilized for each database are provided in Online Supplementary Data 1. No language restriction was implemented. A manual search for conceivably related articles utilizing references of the included studies was additionally performed. This systematic review was performed following the PRISMA (Preferred Reporting Items for Systematic Reviews and Meta-Analysis) statement [71].

\subsection{Study Selection}

Studies were included in this systematic review if they were clinical trials or observational studies that reported the incidence of AKI (using standard AKI definitions including RIFLE (Risk, Injury, Failure, Loss of kidney function, and End-stage kidney disease) [72], AKIN (Acute Kidney Injury Network) [73], and KDIGO (Kidney Disease: Improving Global Outcomes) classifications) [74], severe AKI requiring renal replacement therapy (RRT), and mortality risk of AKI among adult patients (age $\geq 18$ years old) on ECMO. Eligible studies needed to provide the data to evaluate the incidence or mortality rate of AKI with $95 \%$ confidence intervals (CI). Retrieved articles were independently examined for eligibility by the two authors (C.T. and W.C.). Inconsistencies were discussed and resolved by shared agreement. The size of the study did not limit inclusion. 


\subsection{Data Collection Process}

A structured data collecting form was adopted to gather the following data from individual study including title, name of authors, publication year, year of the study, country where the study was conveyed, type of ECMO, AKI definition, incidence of AKI, incidence of severe AKI requiring RRT, and mortality risk of AKI among patients on ECMO.

\subsection{Statistical Analysis}

We used the Comprehensive Meta-Analysis software version 3.3.070 (Biostat Inc, Englewood, NJ, USA) to conduct the meta-analysis. Adjusted point estimates of included studies were consolidated by the generic inverse variance method of DerSimonian-Laird, which assigned the weight of individual study based on its variance [75]. Due to the probability of between-study variance, we applied a random-effects model to pool outcomes of interest, including the incidence of AKI and mortality risk. Statistical heterogeneity of studies was assessed by the Cochran's $Q$ test $(p<0.05$ for a statistical significance) and the $I^{2}$ statistic ( $\leq 25 \%$ : insignificant heterogeneity, $26 \%-50 \%$ : low heterogeneity, $51 \%-75 \%$ : moderate heterogeneity and $\geq 75 \%$ : high heterogeneity) [76]. The presence of publication bias was evaluated by both the funnel plot and the Egger test [77].

\section{Results}

A total of 1,632 potentially eligible articles were identified with our search approach. After excluding 644 articles that were either in-vitro studies, focused on pediatric patient population, animal studies, case reports, correspondences, or review articles, and 831 articles due to being duplicates, 157 articles remained for full-length article review. Seventy-three articles were subsequently excluded as they did not provide data on the incidence of AKI or mortality of AKI, while 33 articles were excluded because they were not clinical trials or observational studies. Ten studies [19-28] were additionally excluded because they did not use a standard AKI definition or did not report the incidence of severe AKI requiring RRT. Therefore, 41 cohort studies [7,15,29-67] with a total of 10,282 adult patients receiving ECMO were enrolled. The systematic review of the literature flowchart is demonstrated in Figure 1. The characteristics of the included studies are shown in Table 1. 
Potentially relevant articles identified from search of MEDLINE,

EMBASE, and Cochrane Database of Systematic Reviews

$$
(\mathrm{n}=1,632)
$$

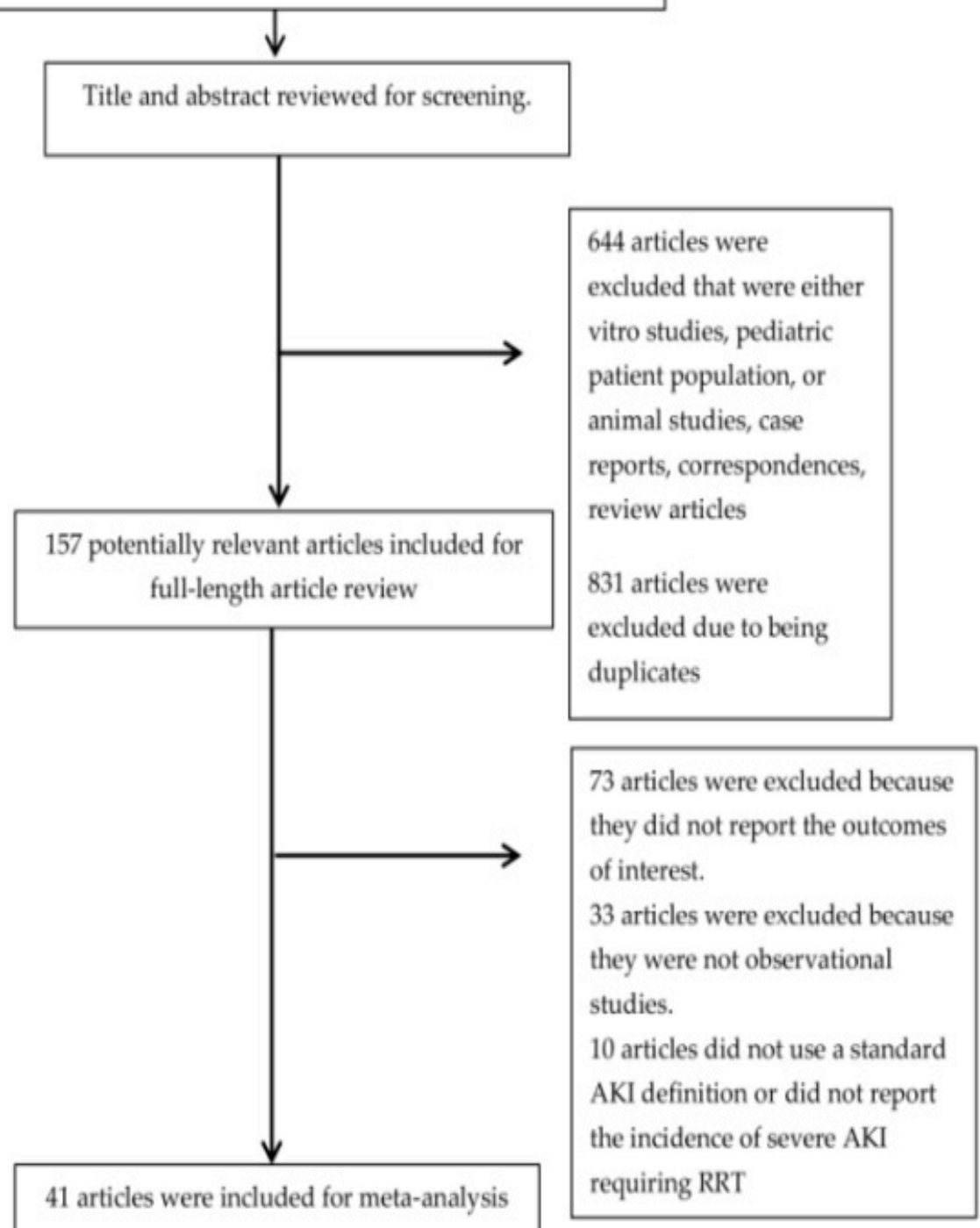

Figure 1. The flowchart for the systematic review. 
Table 1. Main characteristic of studies included in this meta-analysis of AKI incidence and mortality among patients requiring ECMO [7,15,29-67].

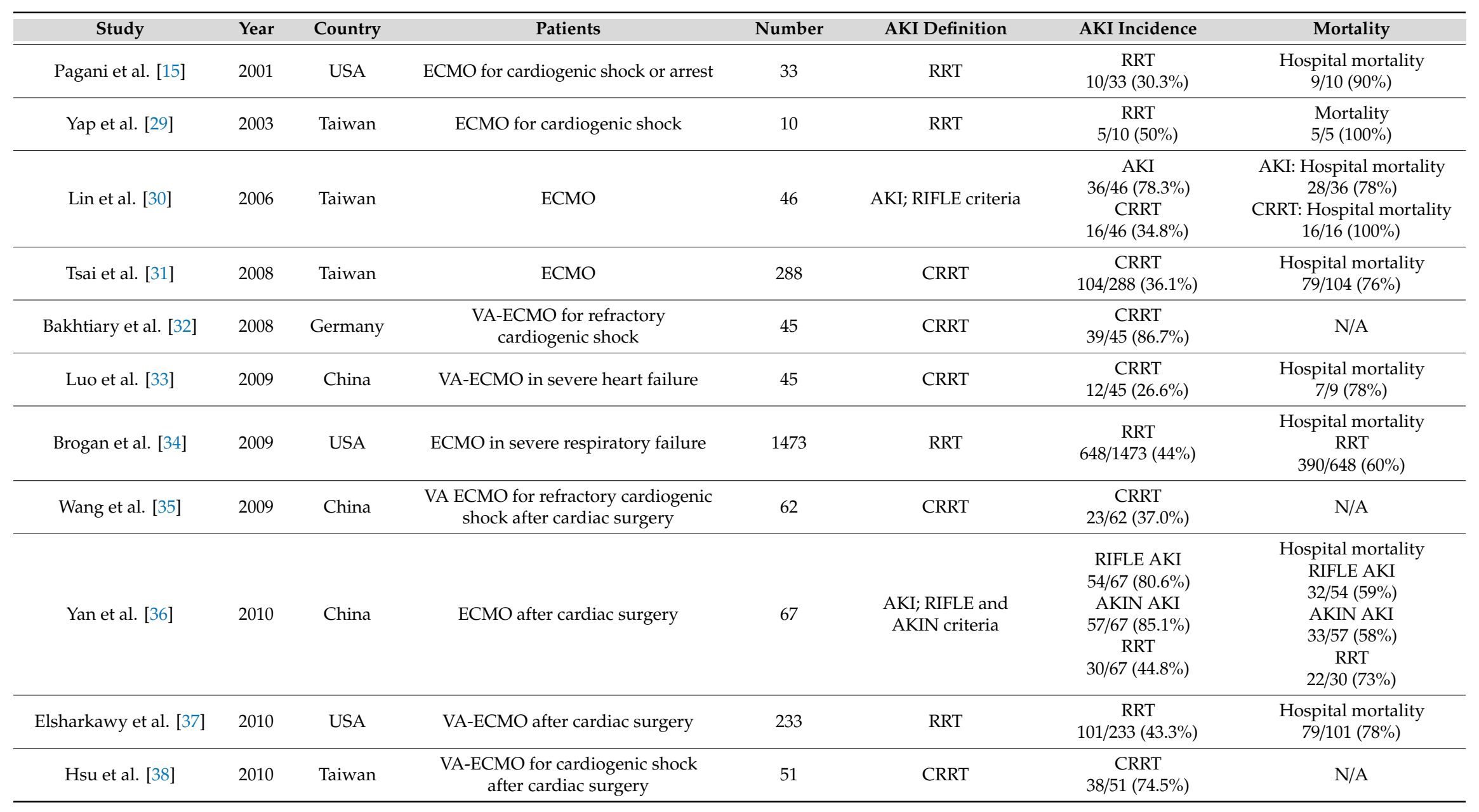


Table 1. Cont

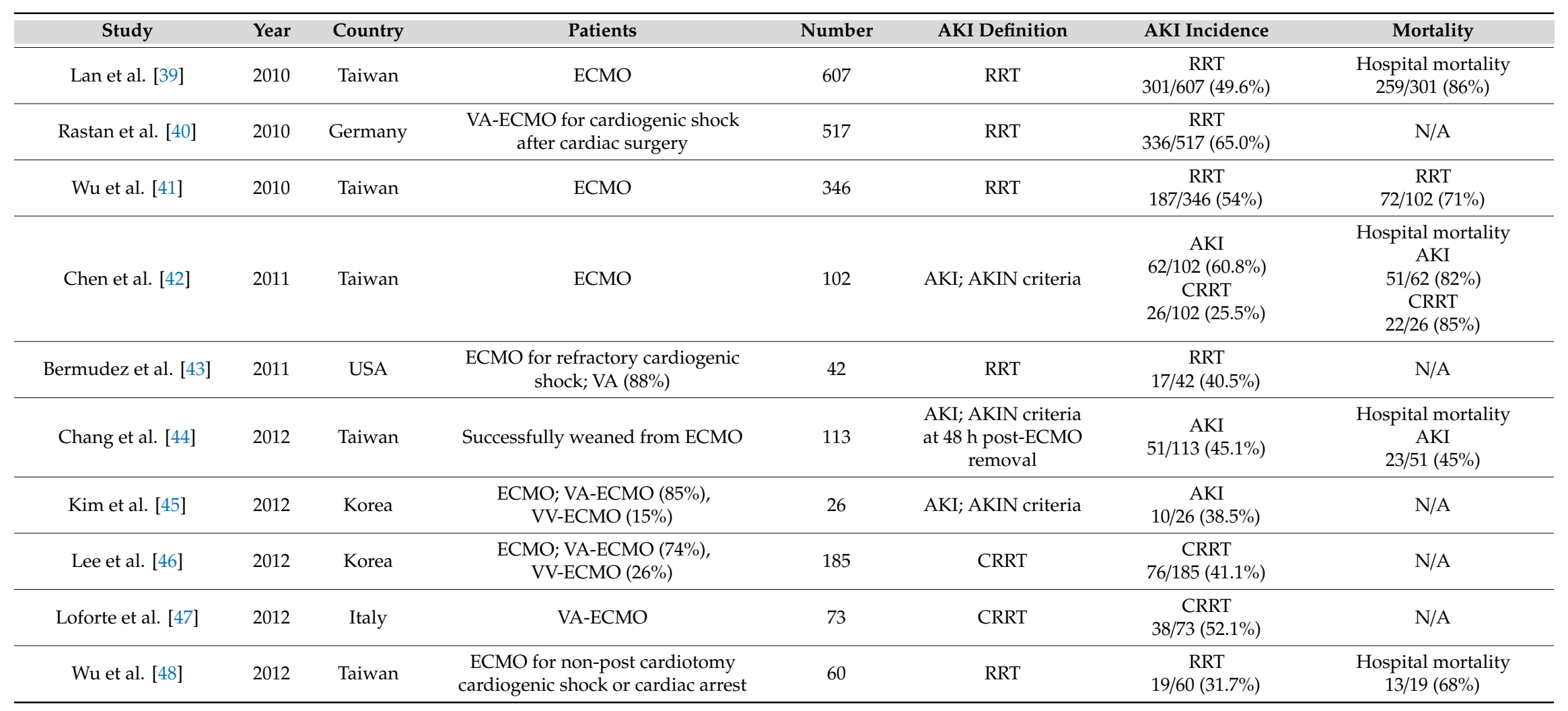


Table 1. Cont.

\begin{tabular}{|c|c|c|c|c|c|c|c|}
\hline Study & Year & Country & Patients & Number & AKI Definition & AKI Incidence & Mortality \\
\hline Aubron et al. [49] & 2013 & Australia & $\begin{array}{l}\text { ECMO; VA-ECMO (67\%), } \\
\text { VV-ECMO }(33 \%)\end{array}$ & 158 & RRT & $\begin{array}{c}\text { VA-ECMO } \\
\text { RRT } \\
\text { 61/105 (58.1\%) } \\
\text { VV-ECMO } \\
\text { RRT } \\
\text { 27/53 }(50.9 \%)\end{array}$ & $\begin{array}{c}\text { Hospital mortality } \\
\text { VA-ECMO } \\
\text { RRT } \\
27 / 61(44 \%) \\
\text { VV-ECMO } \\
\text { RRT } \\
13 / 27(48 \%)\end{array}$ \\
\hline Kielstein et al. [50] & 2013 & Germany & $\begin{array}{l}\text { ECMO; VA-ECMO }(45 \%) \\
\text { VV-ECMO }(55 \%)\end{array}$ & 200 & RRT & $\begin{array}{c}\text { RRT } \\
\text { 117/200 (58.5\%) } \\
\text { RRT after ECMO } \\
\text { 92/175 (52.6\%) } \\
\end{array}$ & $\begin{array}{l}\text { 90-day mortality } \\
\text { 97/117 (83\%) }\end{array}$ \\
\hline Wu et al. [51] & 2013 & Taiwan & $\begin{array}{l}\text { ECMO for acute myocardial } \\
\text { infarction-induced cardiac arrest }\end{array}$ & 35 & RRT & $\begin{array}{c}\text { RRT } \\
16 / 35(45.7 \%)\end{array}$ & $\begin{array}{c}\text { Hospital mortality } \\
14 / 16(88 \%)\end{array}$ \\
\hline Lazzeri et al. [52] & 2013 & Italy & ECMO for refractory cardiac arrest & 25 & RRT & $\begin{array}{c}\text { RRT } \\
16 / 24(66.7 \%)\end{array}$ & $\begin{array}{l}\text { Mortality } \\
9 / 16(56 \%)\end{array}$ \\
\hline Unosawa et al. [53] & 2013 & Japan & $\begin{array}{l}\text { VA-ECMO for refractory cardiogenic } \\
\text { shock after cardiac surgery }\end{array}$ & 47 & RRT & $\begin{array}{c}\text { RRT } \\
15 / 47(31.9 \%) \\
\end{array}$ & $\begin{array}{c}\text { Mortality on ECMO } \\
7 / 15(46.7 \%) \\
\end{array}$ \\
\hline Xue et al. [54] & 2014 & China & ECMO in lung transplantation & 45 & AKI; AKIN criteria & $\begin{array}{c}\text { AKI } \\
17 / 45(37.8 \%) \\
\end{array}$ & N/A \\
\hline Schmidt et al. [7] & 2014 & Australia & $\begin{array}{l}\text { ECMO for refractory cardiogenic } \\
\text { shock or acute respiratory failure }\end{array}$ & 172 & AKI; RIFLE criteria & $\begin{array}{c}\text { AKI at ECMO day } 1 \\
\text { 98/172 (57.0\%) } \\
\text { CRRT during ECMO } \\
103 / 172(59.9 \%)\end{array}$ & $\begin{array}{c}\text { 90-day mortality } \\
\text { CRRT } \\
34 / 103(33 \%)\end{array}$ \\
\hline Hsiao et al. [55] & 2014 & Taiwan & ECMO for ARDS & 81 & CRRT & $\begin{array}{c}\text { CRRT } \\
33 / 81(40.7 \%)\end{array}$ & $\begin{array}{c}\text { Hospital mortality } \\
\text { CRRT } \\
22 / 33(67 \%)\end{array}$ \\
\hline Lee et al. [56] & 2015 & Korea & $\begin{array}{l}\text { ECMO; VA-ECMO (71\%), } \\
\text { VV-ECMO }(29 \%)\end{array}$ & 322 & AKI; KDIGO criteria & $\begin{array}{c}\text { AKI } \\
265 / 322(82.3 \%)\end{array}$ & $\begin{array}{l}\text { Hospital mortality } \\
151 / 265(57 \%)\end{array}$ \\
\hline Haneya [57] & 2015 & Germany & VV-ECMO for ARDS & 262 & AKI; KDIGO criteria & $\begin{array}{c}\text { AKI } \\
\text { 109/262 (41.6\%) } \\
\text { RRT during ECMO } \\
\text { 52/262 (19.8\%) }\end{array}$ & $\begin{array}{c}\text { Mortality } \\
\text { AKI } \\
\text { 56/109 (51\%) } \\
\text { RRT during ECMO } \\
\text { 23/52 (44\%) }\end{array}$ \\
\hline
\end{tabular}


Table 1. Cont

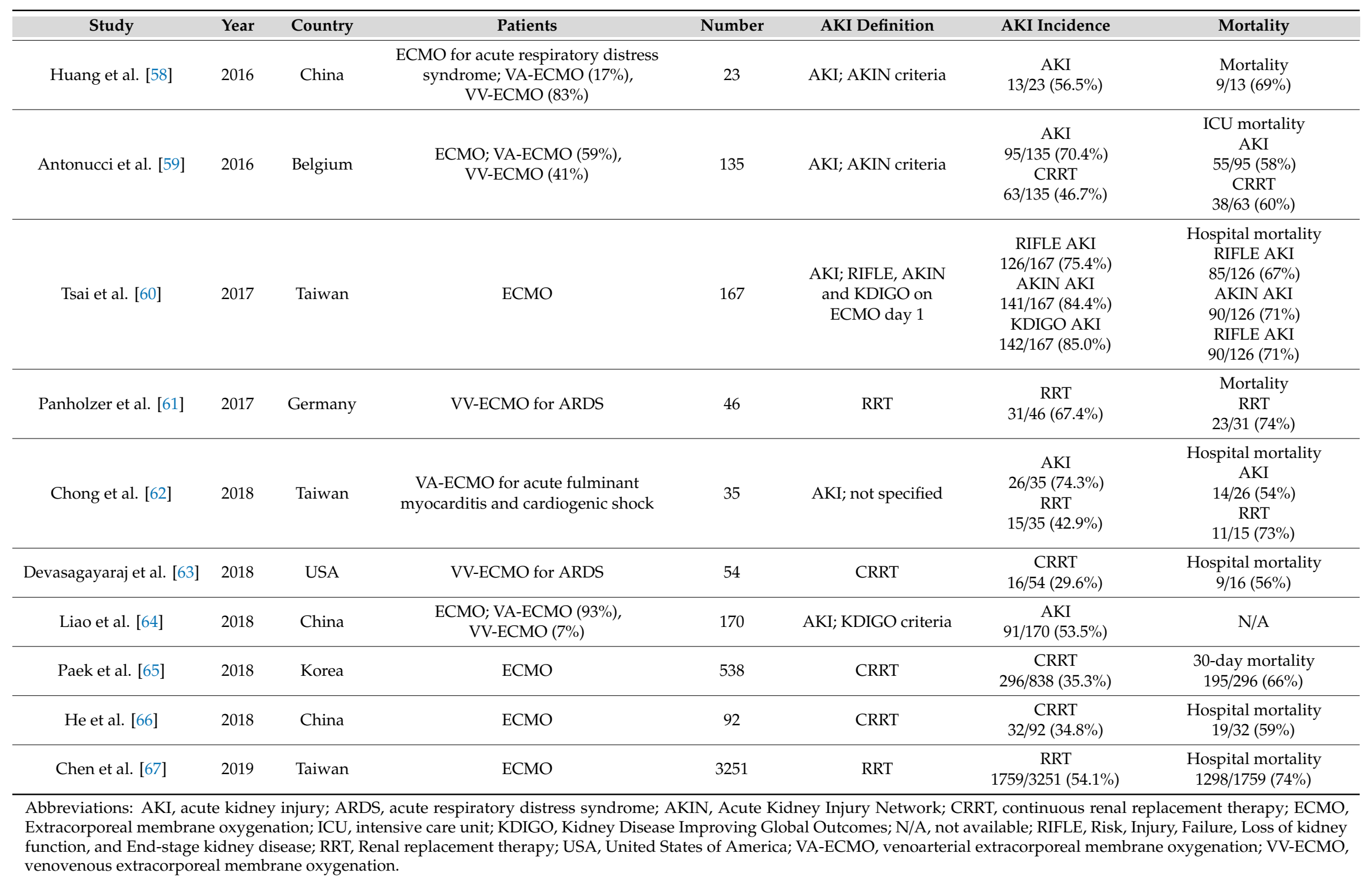




\subsection{Incidence of AKI in Patients Requiring ECMO}

Overall, the pooled estimated incidence of AKI and severe AKI requiring RRT while on ECMO were $62.8 \%$ (95\%CI: $52.1 \%-72.4 \%, I^{2}=94 \%$, Figure 2 A) and $44.9 \%$ (95\%CI: $40.8 \%-49.0 \%, I^{2}=91 \%$, Figure 2B), respectively. Subgroup analyses were performed according to AKI definitions. The pooled estimated incidence rates of AKI by RIFLE, AKIN, and KDIGO criteria were $67.5 \%(95 \% \mathrm{CI}$ : $\left.43.9 \%-84.6 \%, I^{2}=85 \%\right), 57.8 \%$ (95\%CI: $\left.44.6 \%-70.0 \%, I^{2}=86 \%\right)$, and $68.2 \%\left(95 \% C I: 43.8 \%-85.55 \%, I^{2}=\right.$ $98 \%)$, respectively.
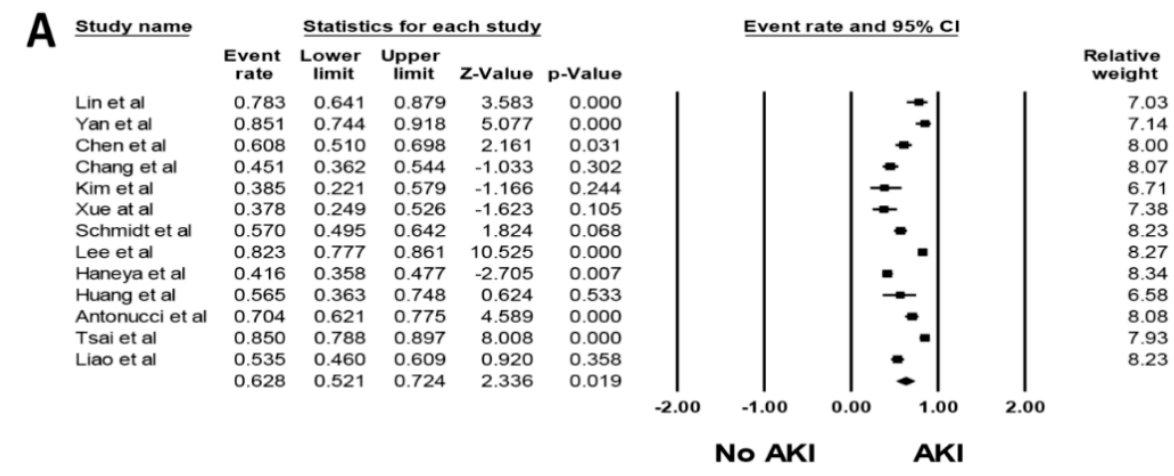

B
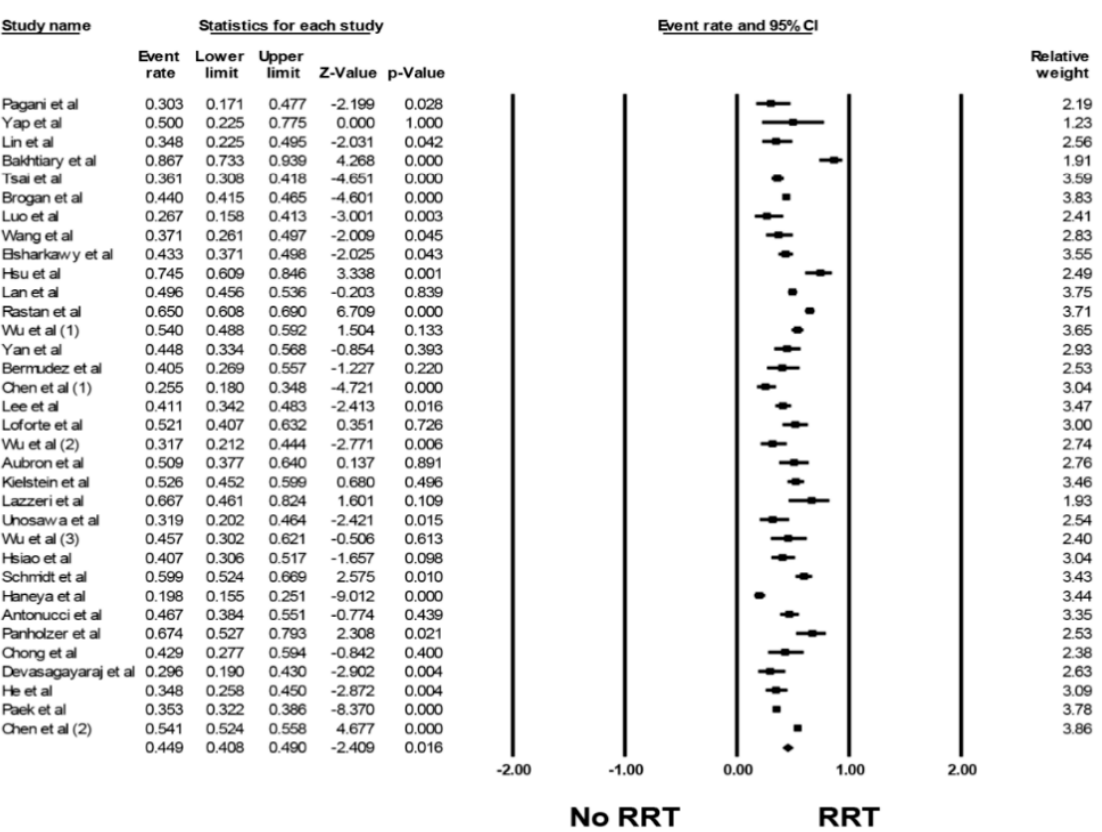

Figure 2. Forest plots of the included studies assessing (A) incidence rates of AKI while on ECMO and (B) incidence rate of severe AKI requiring RRT while on ECMO. A diamond data marker depicts the overall rate from each included study (square data marker) and 95\%CI.

Subgroup analysis based on the type of ECMO was also performed. Pooled estimated incidence of AKI and severe AKI requiring RRT while on venoarterial (VA)-ECMO were 60.8\% (95\%CI: 32.9\%-83.1\%, $I^{2}=96 \%$ ) and $49.5 \%$ (95\%CI: 39.6\%-59.4\%, $I^{2}=90 \%$ ), respectively. Pooled estimated incidence of AKI and severe AKI requiring RRT while on venovenous (VV)-ECMO were 45.7\% (95\%CI: 33.2\%-58.8\%, $I^{2}=47 \%$ ) and $37.0 \%$ (95\%CI: $\left.14.8 \%-66.5 \%, I^{2}=95 \%\right)$, respectively. Meta-regression showed that year of the study did not significantly affect the incidence of AKI $(p=0.67)$ or AKI requiring RRT $(p=0.83)$, as shown in Figure 3. 
A

Regression of Logit event rate on Year of Study

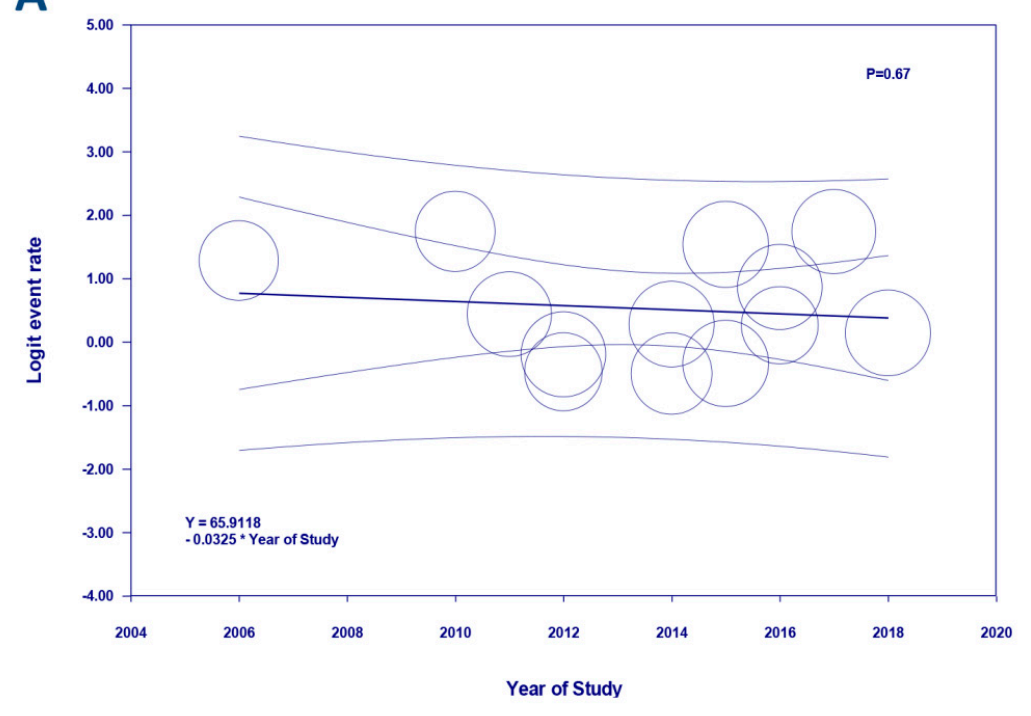

B

Regression of Logit event rate on Year of Study

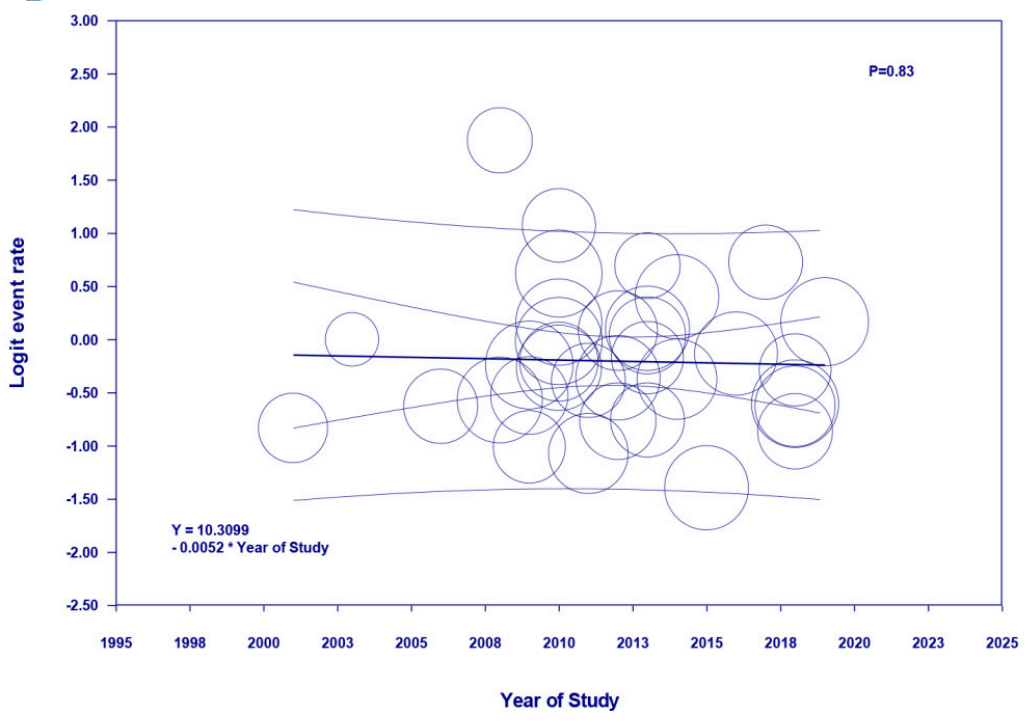

Figure 3. Meta-regression analyses showed that year of the study did not significantly affect (A) the incidence of AKI ( $p=0.67$ ) or (B) AKI requiring RRT ( $p=0.83$ ). The solid black line depicts the weighted regression line based on variance-weighted least squares. The inner and outer lines represent the $95 \% \mathrm{CI}$ and prediction interval encompassing the regression line. The circles indicate log event rates in individual study.

\subsection{AKI associated Mortality in Patients Requiring ECMO}

Mortality rate and mortality risk associated with AKI in patients requiring ECMO are demonstrated in Tables 1 and 2, respectively. The pooled estimated hospital and/or 90-day mortality rates of patients with AKI and severe AKI requiring RRT while on ECMO were $62.0 \%$ (95\%CI: $54.7 \%-68.8 \%, I^{2}=73 \%$, Figure $4 \mathrm{~A}$ ) and $68.4 \%$ (95\%CI: $62.6 \%-73.6 \%, I^{2}=87 \%$, Figure $\left.4 \mathrm{~B}\right)$, respectively. 
Table 2. Characteristics of studies included in this meta-analysis of AKI associated mortality risk among patients requiring ECMO.

\begin{tabular}{|c|c|c|c|c|}
\hline Study. & Year & Number & Outcomes & Confounder Adjustment \\
\hline Pagani et al. [15] & 2001 & 33 & $\begin{array}{l}\text { Hospital mortality } \\
8.25(0.89-76.12)\end{array}$ & None \\
\hline Lin et al. [30] & 2006 & 46 & $\begin{array}{l}\text { Hospital mortality } \\
\text { AKI: } 14.0 \text { (2.46-79.55) } \\
\text { CRRT: } 16 / 16 \text { vs. } 14 / 30\end{array}$ & None \\
\hline Luo et al. [33] & 2009 & 45 & $\begin{array}{l}\text { Hospital mortality } \\
\text { CRRT: } 7.0 \text { (1.26-38.99) }\end{array}$ & None \\
\hline Brogan et al. [34] & 2009 & 1473 & $\begin{array}{c}\text { Hospital mortality } \\
\text { Renal insufficiency/failure: } 2.13 \text { (1.69-2.72) } \\
\text { RRT: } 2.13 \text { (1.73-2.63) }\end{array}$ & $\begin{array}{l}\text { Age, duration of mechanical ventilation, } \\
\text { weight, pre-ECMO pH, race, diagnosis, ECMO } \\
\text { mode, post-ECMO complication }\end{array}$ \\
\hline Elsharkawy et al. [37] & 2010 & 233 & $\begin{array}{l}\text { Hospital mortality } \\
\text { RRT: } 3.18 \text { (1.77-5.70) }\end{array}$ & None \\
\hline Yan et al. [36] & 2010 & 67 & $\begin{array}{c}\text { Hospital mortality } \\
\text { RIFLE AKI: } 8.0(1.61-39.68) \\
\text { AKIN AKI: } 12.38(1.47-104.33) \\
\text { CRRT: } 5.73(1.98-16.58)\end{array}$ & None \\
\hline Lan et al. [39] & 2010 & 607 & $\begin{array}{l}\text { Hospital mortality } \\
\text { RRT: } 6.49 \text { (4.12-10.23) }\end{array}$ & $\begin{array}{l}\text { Age, stroke, pre-ECMO infection, } \\
\text { hypoglycemia, alkalosis }\end{array}$ \\
\hline Chen et al. [67] & 2011 & 102 & $\begin{array}{l}\text { Hospital mortality } \\
\text { AKI: } 4.32 \text { (1.65-11.30) } \\
\text { CRRT: } 5.80 \text { (1.82-18.43) }\end{array}$ & Age, GCS \\
\hline Chang et al. [44] & 2012 & 113 & $\begin{array}{l}\text { Hospital mortality } \\
\text { AKI: } 2.1(1.48-3.00)\end{array}$ & None \\
\hline Wu et al. [48] & 2012 & 60 & $\begin{array}{c}\text { Hospital mortality } \\
\text { RRT: } 3.76 \text { (1.18-11.95) }\end{array}$ & None \\
\hline Kielstein et al. [50] & 2013 & 200 & $\begin{array}{l}\text { 90-day mortality } \\
\text { RRT: } 5.47(2.87-10.44)\end{array}$ & None \\
\hline
\end{tabular}


Table 2. Cont

\begin{tabular}{|c|c|c|c|c|}
\hline Study. & Year & Number & Outcomes & Confounder Adjustment \\
\hline Aubron et al. [49] & 2013 & 158 & $\begin{array}{c}\text { VA ECMO } \\
\text { RRT: } 2.12(0.92-4.88) \\
\text { VV ECMO } \\
\text { RRT: } 2.52(0.80-7.95)\end{array}$ & None \\
\hline Wu et al. [51] & 2013 & 35 & $\begin{array}{l}\text { Hospital mortality } \\
\text { RRT: } 12(2.08-69.09)\end{array}$ & None \\
\hline Slottosch et al. [23] & 2013 & 77 & $\begin{array}{l}\text { 30-day mortality } \\
\text { Renal failure: } 2.20(0.78-6.12)\end{array}$ & None \\
\hline Unosawa et al. [53] & 2013 & 47 & $\begin{array}{l}\text { Mortality during ECMO } \\
\text { RRT: } 1.67(0.48-5.83)\end{array}$ & None \\
\hline Lazzeri et al. [52] & 2013 & 25 & $\begin{array}{c}\text { Mortality } \\
\text { RRT: } 2.14(0.38-12.20)\end{array}$ & None \\
\hline Hsiao et al. [55] & 2014 & 81 & $\begin{array}{l}\text { Hospital mortality } \\
\text { CRRT: } 2.17(0.87-5.45)\end{array}$ & None \\
\hline Schmidt et al. [7] & 2014 & 172 & $\begin{array}{c}\text { Hospital mortality } \\
\text { CRRT at ECMO day 1-3: } 4.1 \text { (1.71-9.82) } \\
\text { 90-day mortality } \\
\text { CRRT at ECMO day 1-3: } 3.17 \text { (1.32-7.61) }\end{array}$ & $\begin{array}{l}\text { APACHE, fluid balance, major bleeding, } \\
\text { propensity score }\end{array}$ \\
\hline Lee et al. [56] & 2015 & 322 & $\begin{array}{l}\text { Hospital mortality } \\
\text { AKI: } 3.71 \text { (1.96-7.02) }\end{array}$ & None \\
\hline Haneya et al. [57] & 2015 & 262 & $\begin{array}{c}\text { Mortality } \\
\text { AKI: } 2.18(1.31-3.61) \\
\text { RRT during ECMO: } 1.72(0.53-5.59)\end{array}$ & $\begin{array}{l}\text { Age, SOFA score, minute volume, } \mathrm{pH} \text {, lactate, } \\
\text { RRT prior to ECMO, RBC, and FFP transfusion }\end{array}$ \\
\hline Huang et al. [58] & 2016 & 23 & $\begin{array}{c}\text { Mortality } \\
\text { AKI: } 20.25(1.88-218.39)\end{array}$ & None \\
\hline
\end{tabular}


Table 2. Cont.

\begin{tabular}{|c|c|c|c|c|}
\hline Study. & Year & Number & Outcomes & Confounder Adjustment \\
\hline Lyu et al. [27] & 2016 & 84 & $\begin{array}{c}\text { Mortality } \\
\text { ARF: } 23.90(7.00-81.60)\end{array}$ & None \\
\hline Antonucci et al. [59] & 2016 & 135 & $\begin{array}{c}\text { ICU mortality } \\
\text { AKI: } 1.86(0.88-3.93) \\
\text { CRRT: } 1.70(0.85-3.37)\end{array}$ & None \\
\hline Tsai et al. [60] & 2017 & 167 & $\begin{array}{c}\text { Hospital mortality } \\
\text { RIFLE AKI: } 8.55(3.63-20.16) \\
\text { AKIN AKI: } 13.53(3.87-47.28) \\
\text { KDIGO AKI: } 12.69(3.62-44.46)\end{array}$ & None \\
\hline Panholzer et al. [61] & 2017 & 46 & $\begin{array}{c}\text { Mortality } \\
\text { RRT: } 40.25(4.54-356.93)\end{array}$ & None \\
\hline Martucci et al. [28] & 2017 & 82 & $\begin{array}{c}\text { Mortality on ECMO } \\
\text { AKI stage 3: } 4.55 \text { (1.37-15.17) }\end{array}$ & None \\
\hline Chong et al. [62] & 2018 & 35 & $\begin{array}{l}\text { Hospital mortality } \\
\text { AKI: } 9.33(1.02-85.70) \\
\text { RRT: } 11.0(2.26-53.64)\end{array}$ & None \\
\hline Devasagayaraj et al. [63] & 2018 & 54 & $\begin{array}{l}\text { Hospital mortality } \\
\text { RRT: } 5.69(1.58-20.56)\end{array}$ & None \\
\hline Chen et al. [67] & 2019 & 3,251 & $\begin{array}{l}\text { Hospital mortality } \\
\text { RRT: } 3.92(3.36-4.57)\end{array}$ & $\begin{array}{l}\text { Age, sex, ECMO indication, comorbid } \\
\text { conditions, hospital level, study year }\end{array}$ \\
\hline
\end{tabular}

Abbreviations: AKI, acute kidney injury; ARDS, acute respiratory distress syndrome; AKIN, Acute Kidney Injury Network; APACHE, Acute Physiology and Chronic Health Evaluation; CRRT, continuous renal replacement therapy; ECMO, Extracorporeal membrane oxygenation; FFP, fresh frozen plasma; GCS, Glasgow Coma Scale/Score; ICU, intensive care unit; KDIGO, Kidney Disease Improving Global Outcomes; N/A, not available; RIFLE, Risk, Injury, Failure, Loss of kidney function, and End-stage kidney disease; RBC, red blood cells; RRT, Renal replacement therapy; $\mathrm{pH}$, potential hydrogen; SOFA, Sequential Organ Failure Assessment; VA-ECMO, venoarterial extracorporeal membrane oxygenation; VV-ECMO, venovenous extracorporeal membrane oxygenation. 


\begin{tabular}{|c|c|c|c|c|c|}
\hline \multirow[t]{2}{*}{ A Study name } & \multicolumn{5}{|c|}{ Statistics for each study } \\
\hline & $\begin{array}{l}\text { Event } \\
\text { rate }\end{array}$ & $\begin{array}{c}\text { Lower } \\
\text { limit }\end{array}$ & $\begin{array}{c}\text { Upper } \\
\text { limit }\end{array}$ & Z-Value & p-Value \\
\hline Lin et al & 0.778 & 0.615 & 0.885 & 3.125 & 0.002 \\
\hline Yan et al & 0.579 & 0.448 & 0.699 & 1.187 & 0.235 \\
\hline Chen et al & 0.823 & 0.707 & 0.899 & 4.614 & 0.000 \\
\hline Chang et al & 0.451 & 0.321 & 0.588 & -0.699 & 0.485 \\
\hline Lee et al & 0.570 & 0.509 & 0.628 & 2.265 & 0.023 \\
\hline Haneya et al & 0.514 & 0.421 & 0.606 & 0.287 & 0.774 \\
\hline Huang et al & 0.692 & 0.409 & 0.880 & 1.349 & 0.177 \\
\hline Antonucci et al & 0.579 & 0.478 & 0.674 & 1.532 & 0.125 \\
\hline Tsai et al & 0.714 & 0.629 & 0.786 & 4.646 & 0.000 \\
\hline \multirow[t]{2}{*}{ Chong et al } & 0.538 & 0.350 & 0.716 & 0.392 & 0.695 \\
\hline & 0.620 & 0.547 & 0.688 & 3.207 & 0.001 \\
\hline
\end{tabular}

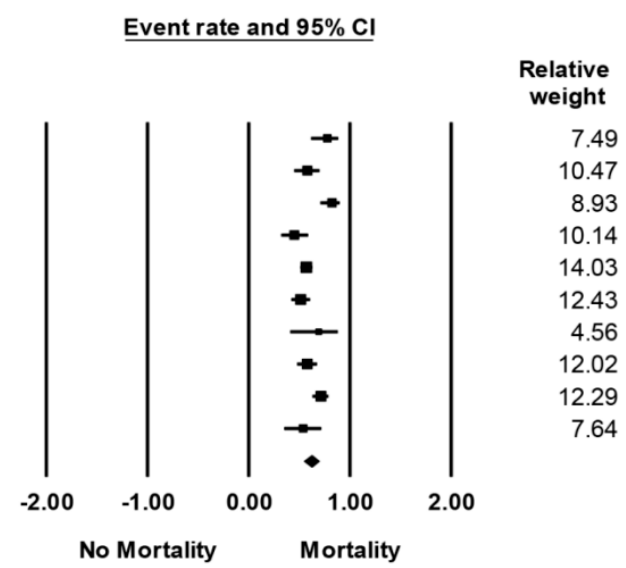

B

\begin{tabular}{lccccc} 
Study name & \multicolumn{5}{c}{ Statistics for each study } \\
& $\begin{array}{l}\text { Event } \\
\text { rate }\end{array}$ & $\begin{array}{c}\text { Lower } \\
\text { limit }\end{array}$ & $\begin{array}{c}\text { Upper } \\
\text { limit }\end{array}$ & Z-Value & p-Value \\
& 0.900 & 0.533 & 0.986 & 2.084 & 0.037 \\
Pagani et al & 0.917 & 0.378 & 0.995 & 1.623 & 0.105 \\
Yap et al & 0.971 & 0.664 & 0.998 & 2.436 & 0.015 \\
Lin et al & 0.760 & 0.668 & 0.832 & 5.014 & 0.000 \\
Tsai et al & 0.778 & 0.421 & 0.944 & 1.562 & 0.118 \\
Luo et al & 0.602 & 0.564 & 0.639 & 5.149 & 0.000 \\
Brogan et al & 0.733 & 0.550 & 0.861 & 2.450 & 0.014 \\
Yan et al & 0.782 & 0.691 & 0.852 & 5.303 & 0.000 \\
Elsharkawy et al & 0.860 & 0.817 & 0.895 & 10.936 & 0.000 \\
Lan et al & 0.706 & 0.611 & 0.786 & 4.029 & 0.000 \\
Wu et al (1) & 0.846 & 0.655 & 0.941 & 3.136 & 0.002 \\
Chen et al (1) & 0.684 & 0.452 & 0.851 & 1.567 & 0.117 \\
Wu et al (2) & 0.455 & 0.354 & 0.559 & -0.852 & 0.394 \\
Aubron et al & 0.829 & 0.750 & 0.887 & 6.430 & 0.000 \\
Kielstein et al & 0.875 & 0.614 & 0.969 & 2.574 & 0.010 \\
Wu et al (3) & 0.563 & 0.324 & 0.775 & 0.499 & 0.618 \\
Lazzeri et al & 0.467 & 0.241 & 0.707 & -0.258 & 0.796 \\
Unosawa et al & 0.463 & 0.246 & 0.426 & -3.378 & 0.001 \\
Schmidt et al & 0.330 & 0.492 & 0.805 & 1.877 & 0.061 \\
Hsiao et al & 0.667 & 0.491 \\
Haneya et al & 0.442 & 0.315 & 0.578 & -0.830 & 0.406 \\
Antonucci et al & 0.603 & 0.479 & 0.716 & 1.626 & 0.104 \\
Panholzer et al & 0.742 & 0.563 & 0.865 & 2.573 & 0.010 \\
Chong et al & 0.733 & 0.467 & 0.896 & 1.733 & 0.083 \\
Devasagayaraj et al & 0.563 & 0.324 & 0.775 & 0.499 & 0.618 \\
Paek et al & 0.659 & 0.603 & 0.711 & 5.366 & 0.000 \\
He et al & 0.594 & 0.419 & 0.747 & 1.054 & 0.292 \\
Chen et al (2) & 0.738 & 0.717 & 0.758 & 19.093 & 0.000 \\
& 0.684 & 0.626 & 0.736 & 5.943 & 0.000 \\
& & & & &
\end{tabular}

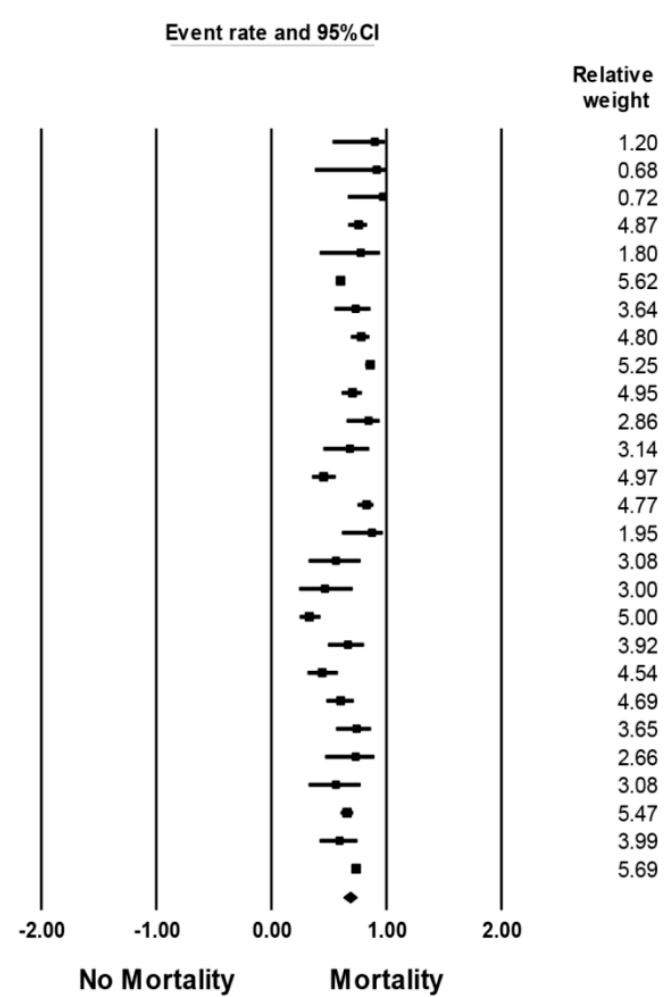

Figure 4. Forest plots of the included studies assessing (A) mortality rate of patients with AKI while on ECMO and (B) mortality rate of patients with severe AKI requiring RRT while on ECMO. A diamond data label serves as the overall rate from each study (square data marker) and 95\%CI.

The pooled OR of hospital mortality among patients receiving ECMO with AKI on RRT was $3.73\left(95 \%\right.$ CI, 2.87-4.85, $I^{2}=62 \%$, Figure 5A). When the analysis was limited to studies with confounder-adjusted analysis, the increased hospital mortality remained significant among patients receiving ECMO with AKI requiring RRT with pooled OR of $3.32\left(95 \% \mathrm{CI}, 2.21-4.99, I^{2}=82 \%\right.$, Figure 5B). 
Study name

Pagani et a

Luo et al

Yan et al

Chen et al (1)

Kielstein et al

Wu et al (1)

Lazzeri et al

Schmidt et al

Antonucci et al

Chong et al

Devasagayaraj et al

Aubron et al

Brogan et al

Elsahrkawy et al

Hsaio et al

Lan et al

Unosawa et al

Wu et al (2)

Panholzer et al

Haneya et al

Chen (2)
Statistics for each study

Odds Lower Upper

ratio limit limit $Z$-Value $p$-Value

$\begin{array}{lllll}8.250 & 0.892 & 76.297 & 1.859 & 0.063\end{array}$

$\begin{array}{lllll}7.000 & 1.258 & 38.939 & 2.222 & 0.026\end{array}$

$\begin{array}{lllll}5.730 & 1.980 & 16.581 & 3.220 & 0.001\end{array}$

$\begin{array}{llllll}5.800 & 1.823 & 18.457 & 2.976 & 0.003\end{array}$

$\begin{array}{lllll}5.470 & 2.868 & 10.433 & 5.158 & 0.000\end{array}$

$\begin{array}{lllll}12.000 & 2.082 & 69.160 & 2.781 & 0.005\end{array}$

$\begin{array}{lllll}2.140 & 0.378 & 12.126 & 0.860 & 0.390\end{array}$

$\begin{array}{lllll}4.100 & 1.711 & 9.825 & 3.164 & 0.002\end{array}$

$\begin{array}{lllll}1.700 & 0.854 & 3.385 & 1.510 & 0.131\end{array}$

$\begin{array}{lllll}11.000 & 2.258 & 53.590 & 2.968 & 0.003\end{array}$

$\begin{array}{lllll}5.690 & 1.577 & 20.526 & 2.656 & 0.008\end{array}$

$\begin{array}{lllll}2.520 & 0.799 & 7.944 & 1.578 & 0.115\end{array}$

$\begin{array}{lllll}2.130 & 1.728 & 2.626 & 7.076 & 0.000\end{array}$

$\begin{array}{lllll}3.180 & 1.772 & 5.707 & 3.878 & 0.000\end{array}$

$\begin{array}{lllll}2.170 & 0.867 & 5.431 & 1.655 & 0.098\end{array}$

$\begin{array}{lllll}6.490 & 4.119 & 10.227 & 8.061 & 0.000\end{array}$

$\begin{array}{lllll}1.670 & 0.479 & 5.820 & 0.805 & 0.421\end{array}$

$\begin{array}{lllll}3.760 & 1.182 & 11.965 & 2.242 & 0.025\end{array}$

$\begin{array}{lllll}40.250 & 4.539 & 356.886 & 3.319 & 0.001\end{array}$

$\begin{array}{lllll}1.720 & 0.530 & 5.586 & 0.902 & 0.367\end{array}$

$\begin{array}{lllll}3.920 & 3.361 & 4.572 & 17.410 & 0.000\end{array}$

$\begin{array}{lllll}3.730 & 2.868 & 4.852 & 9.812 & 0.000\end{array}$
B study name

$\begin{array}{lcccrr} & \begin{array}{c}\text { Odds } \\ \text { ratio }\end{array} & \begin{array}{c}\text { Lower } \\ \text { limit }\end{array} & \begin{array}{c}\text { Upper } \\ \text { limit }\end{array} & \text { Z-Value } & \text { p-Value } \\ \text { Brogan et al } & 2.130 & 1.728 & 2.626 & 7.076 & 0.000 \\ \text { Lan et al } & 6.490 & 4.119 & 10.227 & 8.061 & 0.000 \\ \text { Chen et al (1) } & 5.800 & 1.823 & 18.457 & 2.976 & 0.003 \\ \text { Schmidt et al } & 4.100 & 1.711 & 9.825 & 3.164 & 0.002 \\ \text { Haneya et al } & 1.720 & 0.530 & 5.586 & 0.902 & 0.367 \\ \text { Antonucci et al } & 1.380 & 0.470 & 4.056 & 0.586 & 0.558 \\ \text { Chen et al (2) } & 3.920 & 3.361 & 4.572 & 17.410 & 0.000 \\ & 3.323 & 2.214 & 4.986 & 5.797 & 0.000\end{array}$

Odds ratio and $95 \% \mathrm{Cl}$
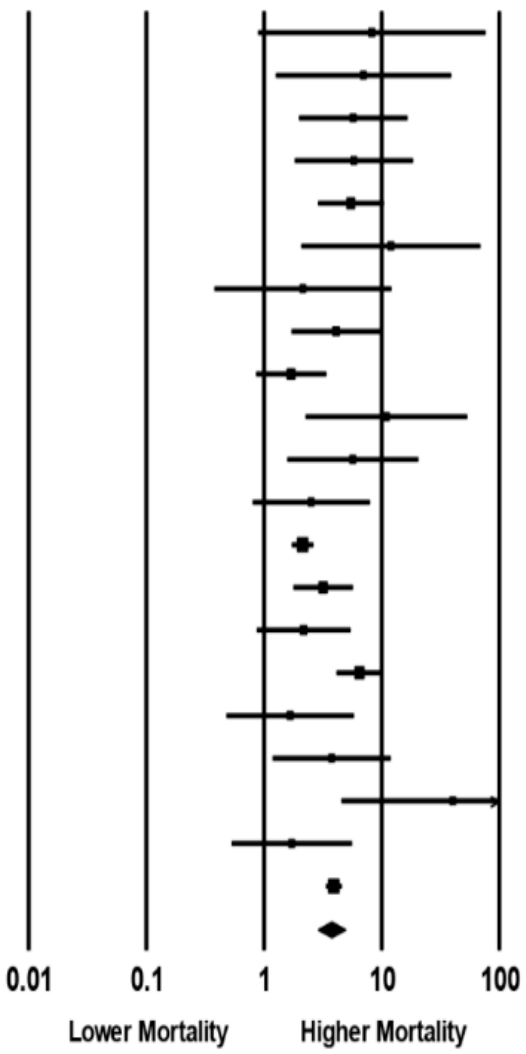

Relative

weight

1.26

1.98

4.11

3.65

7.11

1.91

1.94

5.24

6.72

2.26

3.14

3.69

11.54

7.71

4.95

9.07

3.27

3.65

1.30

3.56

11.94

Higher Mortality

Odds ratio and $95 \% \mathrm{Cl}$

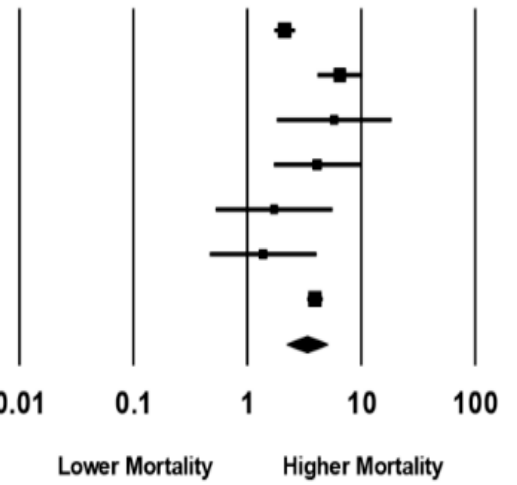

Relative weight

22.40

18.34

8.11

11.32

7.92

8.89

23.03

\begin{tabular}{ccccc}
0.01 & 0.1 & 1 & 10 & 100 \\
& Lower Mortality & \multicolumn{3}{c}{ Higher Mortality }
\end{tabular}

Figure 5. Forest plots of the included studies assessing (A) hospital mortality among patients receiving ECMO with AKI on RRT and (B) hospital mortality among patients receiving ECMO with AKI on RRT limited to studies with confounder-adjusted analysis. A diamond data label serves as the overall rate from each included study (square data marker) and 95\%CI.

Meta-regression showed that year of the study did not significantly affect hospital mortality among patients receiving ECMO with AKI requiring RRT ( $p=0.86)$, as shown in Figure 6. 


\section{Regression of Log odds ratio on Year of Study}

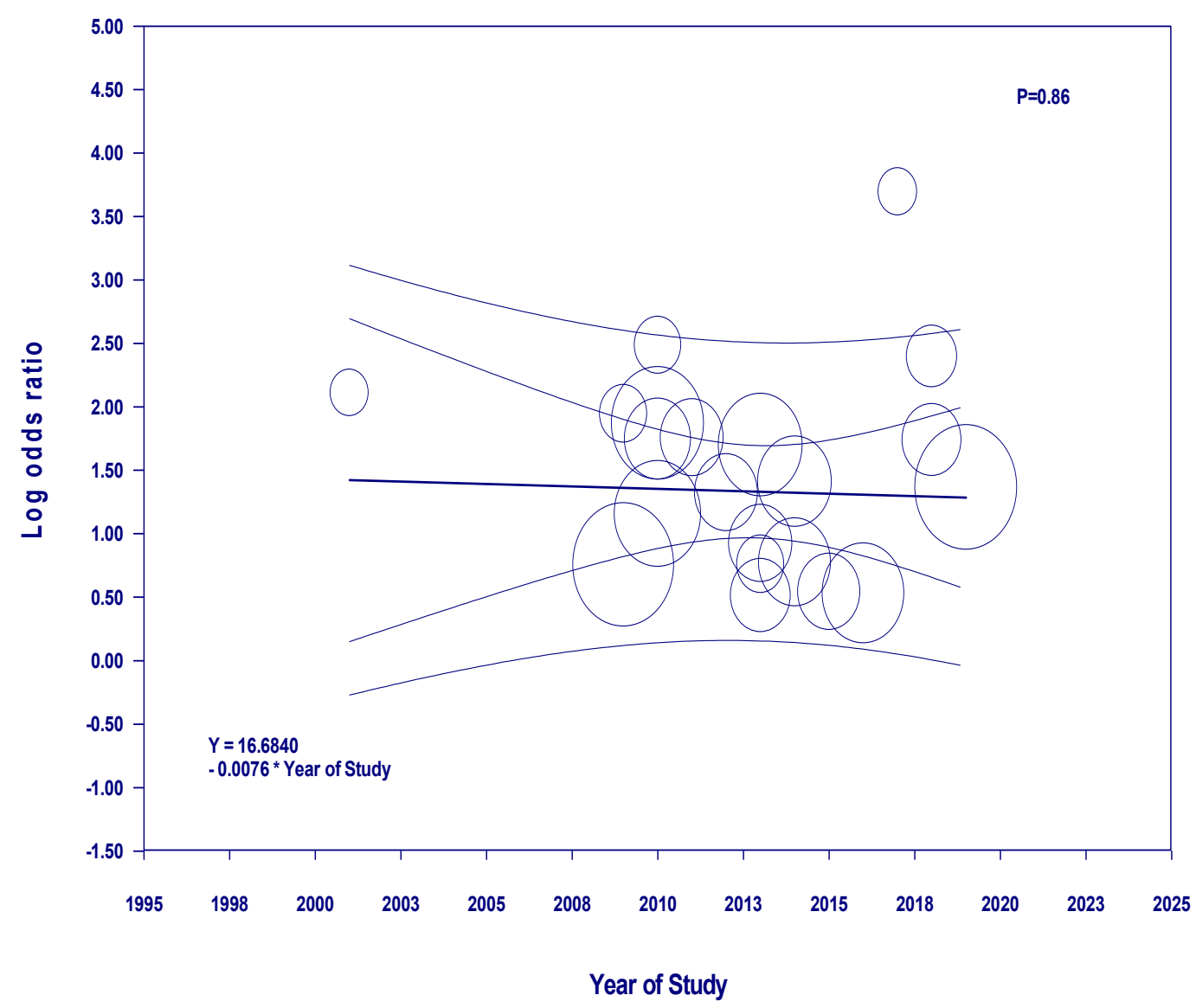

Figure 6. Meta-regression analyses showed that year of the study did not significantly affect hospital mortality among patients receiving ECMO with AKI requiring RRT ( $p=0.86)$. The solid black line depicts the weighted regression line based on variance-weighted least squares. The inner and outer lines represent the $95 \% \mathrm{CI}$ and prediction interval encompassing the regression line. The circles indicate $\log$ event rates in an individual study.

\subsection{Evaluation for Publication Bias}

Funnel plots (Figure 7) and Egger's regression asymmetry tests were utilized to assess for publication bias in our meta-analyses evaluating the incidence of AKI and severe AKI requiring RRT while on ECMO. There was no publication bias as determined by the funnel plot and Egger's regression asymmetry test with $p=0.62$ and $p=0.17$ for the incidence of AKI and severe AKI requiring RRT, respectively. 

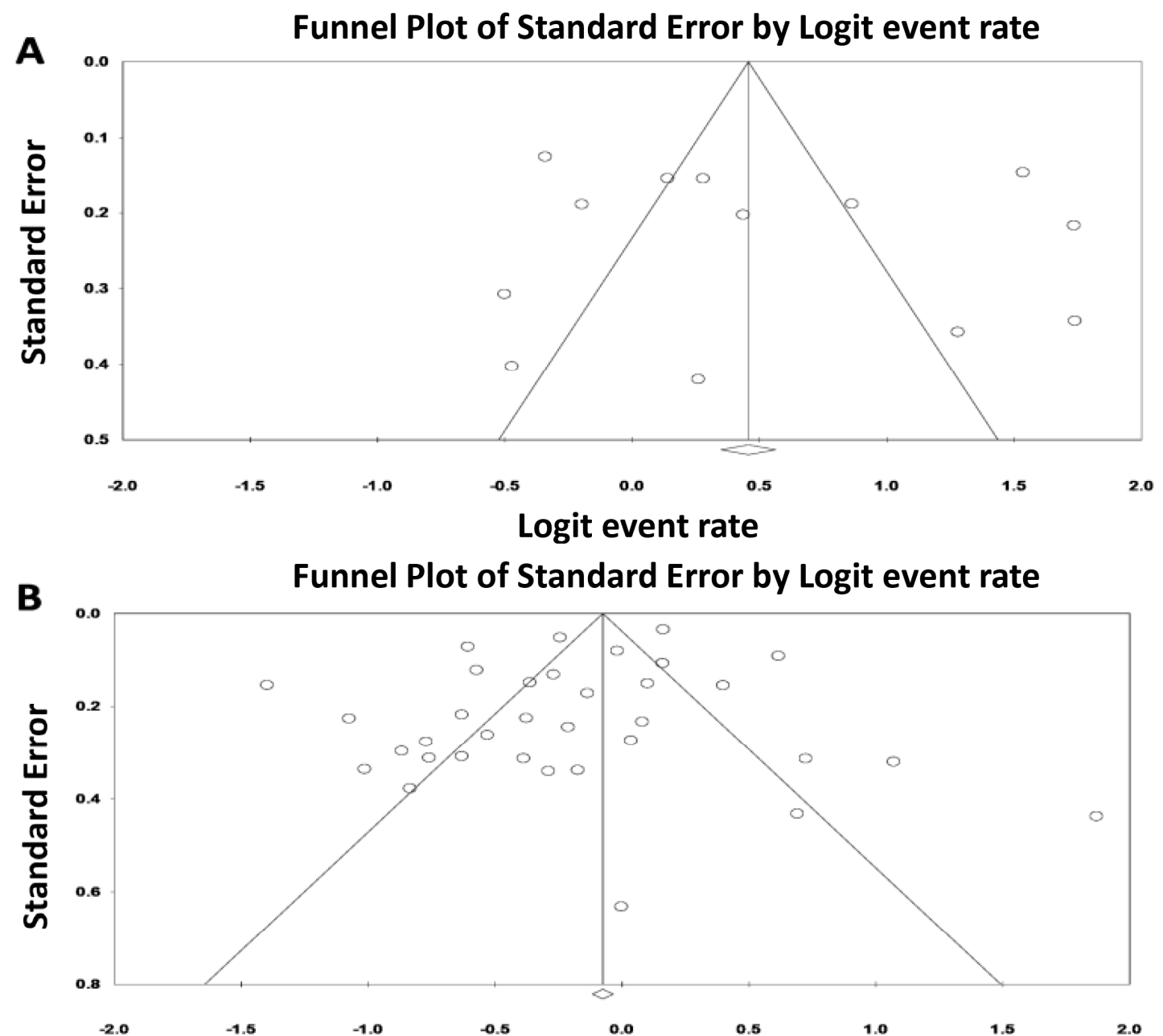

Logit event rate

Figure 7. Funnel plot demonstrated no publication bias in analyses evaluating (A) incidence of AKI in patients requiring ECMO and $(\mathbf{B})$ severe AKI requiring RRT.

\section{Discussion}

The findings of our meta-analysis demonstrate that patients who required ECMO had incidence rates of AKI (using standard AKI definitions) and severe AKI requiring RRT of $62.8 \%$ and $44.9 \%$, respectively. Moreover, patients with AKI and severe AKI requiring RRT had high associated mortality rates of $62.0 \%$ and $68.4 \%$, respectively.

Although the mechanisms underlying ECMO associated-AKI remains unclear, it is likely complex and multifactorial, including contributing factors such as primary disease progression, altered hemodynamics, low cardiac output syndrome, exposure to nephrotoxic agents (for management of underlying diseases), new-onset sepsis, high intrathoracic pressures, fluid overload, ischemia-reperfusion injury, release of proinflammatory mediators and oxidative stress, hemolysis and iron-mediated (hemoglobin-induced) renal injury, and hypercoagulable state resulting in renal microembolisms $[4,8,68,78,79]$. Studies have demonstrated the activation of proinflammatory mediators such as tumor necrosis factor-alpha (TNF- $\alpha$ ), interleukins (e.g., IL-1 $\beta$, IL-6, IL-8) and other cytokine signaling cascades due to the continuous exposure of blood to non-biological and non-endothelialized ECMO interface $[68,80,81]$. Activation of the inflammatory cascades can result in hyperdynamic vasodilated hypotensive states, leading to AKI [68,78]. 
Following the initiation of ECMO treatment, there are improvements in oxygenation and oxygen consumption as well as hemodynamics [3,5-9]. However, ischemia-reperfusion injury can also occur after the restoration of circulation to previously hypoxic cells and hypoperfused organs, leading to the production of reactive oxygen species (ROS) and oxidative stress-mediated injury [68,78]. In addition, ECMO-associated complications or adverse effects such as hemolysis, hemorrhage or thrombosis also can play important roles in the development of AKI [29,68,82-84]. Despite the advance of a new miniaturized ECMO system, hemolysis due to shear stress from the ECMO circuit has been reported among ECMO patients with incidences between 5\% and 18\% [17,85-87]. This can contribute to heme pigment-induced AKI $[83,84]$. Although improvements in the ECMO technology have led to less thrombus development in its circuit with an improved capacity of the circuit to remove large emboli [68,82], smaller thrombi can still develop and result in renal microembolism [68,82], particularly with VA-ECMO [82].

The type of ECMO may also differently affect AKI risk. Our study demonstrated a higher incidence of AKI among patients requiring VA-ECMO (60.8\%) than those requiring VV-ECMO (45.7\%). While VV-ECMO is typically utilized for patients with isolated respiratory failure, VA-ECMO is used for combined severe cardiac and respiratory failure [4]. In VA-ECMO, there is a mixture of pulsatile arterial flow from the native heart and non-pulsatile arterial flow from the ECMO pump. Conversely, VV-ECMO maintains pulsatile cardiac output, and alterations in renal perfusion may conceivably be smaller [4]. Recent studies have shown that pulsatile flow may provide beneficial effects over non-pulsatile flow, especially protective effects on microcirculation and renal perfusion [88-90]. The differences in patient population and pulsatility between the two types of ECMO are likely explanations underlying the higher AKI incidence among patients requiring VA-ECMO.

As there is no treatment available for AKI, management of AKI is limited to appropriate secondary preventive measures and supportive strategies [91-96]. RRT in the form of continuous renal replacement therapy (CRRT) is often required among patients requiring ECMO with severe AKI $[42,55,97]$. Our study demonstrated no significant correlation between the year of study and the incidence of AKI and/or severe AKI requiring RRT despite considerable changes in technology and practice of ECMO among adult patients. Furthermore, we showed a 3.7-fold increased risk of hospital mortality among ECMO patients with severe AKI requiring RRT. Thus, prevention and early identification of AKI among patients at-risk of ECMO-associated AKI could potentially play a crucial role in improved survival. Studies have shown several important AKI risk factors among patients requiring ECMO including older age, elevated lactate levels before ECMO initiation, high dose of inotropic drugs, severely reduced left ventricular ejection fraction, cirrhosis, postcardiotomy shock as an indication for ECMO, and finally ECMO pump speed and its duration $[56,64,67]$. Lee et al. recently observed a lower AKI association with a higher ECMO pump speed [56]. Although the underlying pathophysiology remains unclear, excessive ECMO pump speed has been shown to induce hemolysis and complement activation in vitro and animal model $[98,99]$. In pediatric patients receiving ECMO, Lou et al. also demonstrated higher pump speeds are associated with hemolysis and a number of other adverse clinical outcomes [100]. To prevent hemolysis-mediated kidney injury, it is suggested to limit pump revolutions/min (RPM) to safe levels (i.e., 3000 to $3500 \mathrm{RPM}$ ) in order to avoid excessive negative pressures generated within the pump [101]. Future prospective studies are required to assess the effects of ECMO pump speed on AKI risk in ECMO patients. In addition, future studies creating risk prediction models for ECMO-associated AKI are needed to assist with the prevention of AKI in a timely manner, which could potentially lead to an improvement in patient survival.

Our study has several limitations. Firstly, there are statistical heterogeneities in our meta-analysis. Potential sources for heterogeneities were the variations in patient characteristics among the included studies. However, we performed subgroup analysis to assess the AKI incidence based on types of ECMO and a separate meta-analysis that only included studies with confounder-adjusted analysis for mortality risk. Another limitation was that AKI diagnosis was mainly based on serum creatinine [102-104] while the data on urine output and novel biomarkers for AKI [105-108] were limited. Lastly, this systematic 
review is primarily based on observational studies, as the data from clinical trials or population-based studies were limited. Therefore, it can at best, demonstrate an association but not a causal relationship.

\section{Conclusions}

In conclusion, there is an overall high incidence of AKI and severe AKI requiring RRT in ECMO patients of $62.8 \%$ and $44.9 \%$, respectively. The incidence of ECMO-associated AKI has not changed over time. AKI requiring RRT while on ECMO is associated with 3.7-fold increased risk of hospital mortality. Future studies should focus on strategies for prediction, detection, and prevention of AKI among patients who receive ECMO.

Supplementary Materials: The following are available online at http://www.mdpi.com/2077-0383/8/7/981/s1.

Author Contributions: Conceptualization, C.T., W.C., M.A.M. and K.K.; Data curation, C.T., W.C. and P.L.; Formal analysis, C.T., W.C.; Funding acquisition, K.K.; Investigation, C.T., W.C., P.L. and K.K.; Methodology, C.T., W.C. and K.K.; Project administration, P.L., K.W. and N.S.; Resources, P.L., T.B. and K.W.; Software, K.W.; Supervision, M.A.M. and K.K.; Validation, W.C. and P.L.; Visualization, W.C., T.B. and K.K.; Writing - original draft, C.T. and N.R.A; Writing - review \& editing, C.T., W.C., P.L., N.R.A., T.B., K.W., N.S., M.A.M. and K.K.

Conflicts of Interest: The authors deny any conflict of interest.

\section{References}

1. Guru, P.K.; Singh, T.D.; Passe, M.; Kashani, K.B.; Schears, G.J.; Kashyap, R. Derivation and Validation of a Search Algorithm to Retrospectively Identify CRRT Initiation in the ECMO Patients. Appl. Clin. Inform. 2016, 7, 596-603. [CrossRef] [PubMed]

2. Hill, J.D.; O’Brien, T.G.; Murray, J.J.; Dontigny, L.; Bramson, M.L.; Osborn, J.J.; Gerbode, F. Prolonged extracorporeal oxygenation for acute post-traumatic respiratory failure (shock-lung syndrome). Use of the Bramson membrane lung. N. Engl. J. Med. 1972, 286, 629-634. [CrossRef] [PubMed]

3. Peek, G.J.; Mugford, M.; Tiruvoipati, R.; Wilson, A.; Allen, E.; Thalanany, M.M.; Hibbert, C.L.; Truesdale, A.; Clemens, F.; Cooper, N.; et al. Efficacy and economic assessment of conventional ventilatory support versus extracorporeal membrane oxygenation for severe adult respiratory failure (CESAR): A multicentre randomised controlled trial. Lancet 2009, 374, 1351-1363. [CrossRef]

4. Chen, Y.C.; Tsai, F.C.; Fang, J.T.; Yang, C.W. Acute kidney injury in adults receiving extracorporeal membrane oxygenation. J. Formos. Med. Assoc. 2014, 113, 778-785. [CrossRef] [PubMed]

5. Thiagarajan, R.R.; Barbaro, R.P.; Rycus, P.T.; Mcmullan, D.M.; Conrad, S.A.; Fortenberry, J.D.; Paden, M.L. Extracorporeal Life Support Organization Registry International Report 2016. ASAIO J. 2017, 63, 60-67. [CrossRef] [PubMed]

6. Paden, M.L.; Conrad, S.A.; Rycus, P.T.; Thiagarajan, R.R. Extracorporeal Life Support Organization Registry Report 2012. ASAIO J. 2013, 59, 202-210. [CrossRef]

7. Schmidt, M.; Bailey, M.; Kelly, J.; Hodgson, C.; Cooper, D.J.; Scheinkestel, C.; Pellegrino, V.; Bellomo, R.; Pilcher, D. Impact of fluid balance on outcome of adult patients treated with extracorporeal membrane oxygenation. Intensive Care Med. 2014, 40, 1256-1266. [CrossRef]

8. Hamdi, T.; Palmer, B.F. Review of Extracorporeal Membrane Oxygenation and Dialysis-Based Liver Support Devices for the Use of Nephrologists. Am. J. Nephrol. 2017, 46, 139-149. [CrossRef]

9. Husain-Syed, F.; Ricci, Z.; Brodie, D.; Vincent, J.L.; Ranieri, V.M.; Slutsky, A.S.; Taccone, F.S.; Gattinoni, L.; Ronco, C. Extracorporeal organ support (ECOS) in critical illness and acute kidney injury: From native to artificial organ crosstalk. Intensive Care Med. 2018, 44, 1447-1459. [CrossRef]

10. Bosarge, P.L.; Raff, L.A.; McGwin, G., Jr.; Carroll, S.L.; Bellot, S.C.; Diaz-Guzman, E.; Kerby, J.D. Early initiation of extracorporeal membrane oxygenation improves survival in adult trauma patients with severe adult respiratory distress syndrome. J. Trauma Acute Care Surg. 2016, 81, 236-243. [CrossRef]

11. Combes, A.; Leprince, P.; Luyt, C.E.; Bonnet, N.; Trouillet, J.L.; Léger, P.; Pavie, A.; Chastre, J. Outcomes and long-term quality-of-life of patients supported by extracorporeal membrane oxygenation for refractory cardiogenic shock. Crit. Care Med. 2008, 36, 1404-1411. [CrossRef] [PubMed] 
12. Massetti, M.; Tasle, M.; Le Page, O.; Deredec, R.; Babatasi, G.; Buklas, D.; Thuaudet, S.; Charbonneau, P.; Hamon, M.; Grollier, G.; et al. Back from irreversibility: Extracorporeal life support for prolonged cardiac arrest. Ann. Thorac. Surg. 2005, 79, 178-183. [CrossRef] [PubMed]

13. Shin, T.G.; Choi, J.H.; Jo, I.J.; Sim, M.S.; Song, H.G.; Jeong, Y.K.; Song, Y.B.; Hahn, J.Y.; Choi, S.H.; Gwon, H.C.; et al. Extracorporeal cardiopulmonary resuscitation in patients with inhospital cardiac arrest: A comparison with conventional cardiopulmonary resuscitation. Crit. Care Med. 2011, 39, 1-7. [CrossRef] [PubMed]

14. Bednarczyk, J.M.; White, C.W.; Ducas, R.A.; Golian, M.; Nepomuceno, R.; Hiebert, B.; Bueddefeld, D.; Manji, R.A.; Singal, R.K.; Hussain, F.; et al. Resuscitative extracorporeal membrane oxygenation for in hospital cardiac arrest: A Canadian observational experience. Resuscitation 2014, 85, 1713-1719. [CrossRef] [PubMed]

15. Pagani, F.D.; Aaronson, K.D.; Swaniker, F.; Bartlett, R.H. The use of extracorporeal life support in adult patients with primary cardiac failure as a bridge to implantable left ventricular assist device. Ann. Thorac. Surg. 2001, 71 (Suppl. 3), S77-S81. [CrossRef]

16. Kagawa, E.; Dote, K.; Kato, M.; Sasaki, S.; Nakano, Y.; Kajikawa, M.; Higashi, A.; Itakura, K.; Sera, A.; Inoue, I.; et al. Should we emergently revascularize occluded coronaries for cardiac arrest?: Rapid-response extracorporeal membrane oxygenation and intra-arrest percutaneous coronary intervention. Circulation 2012, 126, 1605-1613. [CrossRef]

17. Zangrillo, A.; Landoni, G.; Biondi-Zoccai, G.; Greco, M.; Greco, T.; Frati, G.; Patroniti, N.; Antonelli, M.; Pesenti, A.; Pappalardo, F. A meta-analysis of complications and mortality of extracorporeal membrane oxygenation. Crit. Care Resusc. 2013, 15, 172-178.

18. Ostermann, M.; Connor, M.; Jr Kashani, K. Continuous renal replacement therapy during extracorporeal membrane oxygenation: Why, when and how? Curr. Opin. Crit. Care 2018, 24, 493-503. [CrossRef]

19. Kagawa, E.; Inoue, I.; Kawagoe, T.; Ishihara, M.; Shimatani, Y.; Kurisu, S.; Nakama, Y.; Dai, K.; Takayuki, O.; Ikenaga, H.; et al. Assessment of outcomes and differences between in- and out-of-hospital cardiac arrest patients treated with cardiopulmonary resuscitation using extracorporeal life support. Resuscitation 2010, 81, 968-973. [CrossRef]

20. Hei, F.; Lou, S.; Li, J.; Yu, K.; Liu, J.; Feng, Z.; Zhao, J.; Hu, S.; Xu, J.; Chang, Q.; et al. Five-year results of 121 consecutive patients treated with extracorporeal membrane oxygenation at Fu Wai Hospital. Artif. Organs 2011, 35, 572-578. [CrossRef]

21. Tsai, T.Y.; Tsai, F.C.; Chang, C.H.; Jenq, C.C.; Hsu, H.H.; Chang, M.Y.; Tian, Y.C.; Hung, C.C.; Fang, J.T.; Yang, C.W.; et al. Prognosis of patients on extracorporeal membrane oxygenation plus continuous arteriovenous hemofiltration. Chang. Gung Med. J. 2011, 34, 636-643. [PubMed]

22. Belle, L.; Mangin, L.; Bonnet, H.; Fol, S.; Santre, C.; Delavenat, L.; Savary, D.; Bougon, D.; Vialle, E.; Dompnier, A.; et al. Emergency extracorporeal membrane oxygenation in a hospital without on-site cardiac surgical facilities. EuroIntervention 2012, 8, 375-382. [CrossRef] [PubMed]

23. Slottosch, I.; Liakopoulos, O.; Kuhn, E.; Deppe, A.C.; Scherner, M.; Madershahian, N.; Choi, Y.H.; Wahlers, T. Outcomes after peripheral extracorporeal membrane oxygenation therapy for postcardiotomy cardiogenic shock: A single-center experience. J. Surg. Res. 2013, 181, e47-e55. [CrossRef] [PubMed]

24. Seco, M.; Forrest, P.; Jackson, S.A.; Martinez, G.; Andvik, S.; Bannon, P.G.; Ng, M.; Fraser, J.F.; Wilson, M.K.; Vallely, M.P. Extracorporeal membrane oxygenation for very high-risk transcatheter aortic valve implantation. Heart Lung Circ. 2014, 23, 957-962. [CrossRef] [PubMed]

25. Saxena, P.; Neal, J.; Joyce, L.D.; Greason, K.L.; Schaff, H.V.; Guru, P.; Shi, W.Y.; Burkhart, H.; Li, Z.; Oliver, W.C.; et al. Extracorporeal Membrane Oxygenation Support in Postcardiotomy Elderly Patients: The Mayo Clinic Experience. Ann. Thorac. Surg. 2015, 99, 2053-2060. [CrossRef] [PubMed]

26. Thajudeen, B.; Kamel, M.; Arumugam, C.; Ali, S.A.; John, S.G.; Meister, E.E.; Mosier, J.M.; Raz, Y.; Madhrira, M.; Thompson, J.; et al. Outcome of patients on combined extracorporeal membrane oxygenation and continuous renal replacement therapy: A retrospective study. Int. J. Artif. Organs 2015, 38, 133-137. [CrossRef] [PubMed]

27. Lyu, L.; Long, C.; Hei, F.; Ji, B.; Liu, J.; Yu, K.; Chen, L.; Yao, J.; Hu, Q.; Hu, J.; et al. Plasma Free Hemoglobin Is a Predictor of Acute Renal Failure During Adult Venous-Arterial Extracorporeal Membrane Oxygenation Support. J. Cardiothorac. Vasc. Anesth. 2016, 30, 891-895. [CrossRef]

28. Martucci, G.; Panarello, G.; Occhipinti, G. Anticoagulation and Transfusions Management in Veno-Venous Extracorporeal Membrane Oxygenation for Acute Respiratory Distress Syndrome: Assessment of Factors Associated With Transfusion Requirements and Mortality. J. Intensive Care Med. 2017, 34, 630-639. [CrossRef] 
29. Yap, H.J.; Chen, Y.C.; Fang, J.T.; Huang, C.C. Combination of continuous renal replacement therapies (CRRT) and extracorporeal membrane oxygenation (ECMO) for advanced cardiac patients. Ren. Fail. 2003, 25, 183-193. [CrossRef]

30. Lin, C.Y.; Chen, Y.C.; Tsai, F.C.; Tian, Y.C.; Jenq, C.C.; Fang, J.T.; Yang, C.W. RIFLE classification is predictive of short-term prognosis in critically ill patients with acute renal failure supported by extracorporeal membrane oxygenation. Nephrol. Dial. Transplant. 2006, 21, 2867-2873. [CrossRef]

31. Tsai, C.W.; Lin, Y.F.; Wu, V.C.; Chu, T.S.; Chen, Y.M.; Hu, F.C.; Wu, K.D.; Ko, W.J. SAPS 3 at dialysis commencement is predictive of hospital mortality in patients supported by extracorporeal membrane oxygenation and acute dialysis. Eur. J. Cardiothorac. Surg. 2008, 34, 1158-1164. [CrossRef]

32. Bakhtiary, F.; Keller, H.; Dogan, S.; Dzemali, O.; Oezaslan, F.; Meininger, D.; Ackermann, H.; Zwissler, B.; Kleine, P.; Moritz, A. Venoarterial extracorporeal membrane oxygenation for treatment of cardiogenic shock: Clinical experiences in 45 adult patients. J. Thorac. Cardiovasc. Surg. 2008, 135, 382-388. [CrossRef] [PubMed]

33. Luo, X.J.; Wang, W.; Hu, S.S.; Sun, H.S.; Gao, H.W.; Long, C.; Song, Y.H.; Xu, J.P. Extracorporeal membrane oxygenation for treatment of cardiac failure in adult patients. Interact. Cardiovasc. Thorac. Surg. 2009, 9, 296-300. [CrossRef] [PubMed]

34. Brogan, T.V.; Thiagarajan, R.R.; Rycus, P.T.; Bartlett, R.H.; Bratton, S.L. Extracorporeal membrane oxygenation in adults with severe respiratory failure: A multi-center database. Intensive Care Med. 2009, 35, 2105-2114. [CrossRef]

35. Wang, J.; Han, J.; Jia, Y.; Zeng, W.; Shi, J.; Hou, X.; Meng, X. Early and intermediate results of rescue extracorporeal membrane oxygenation in adult cardiogenic shock. Ann. Thorac. Surg. 2009, 88, 1897-1903. [CrossRef] [PubMed]

36. Yan, X.; Jia, S.; Meng, X.; Dong, P.; Jia, M.; Wan, J.; Hou, X. Acute kidney injury in adult postcardiotomy patients with extracorporeal membrane oxygenation: Evaluation of the RIFLE classification and the Acute Kidney Injury Network criteria. Eur. J. Cardiothorac. Surg. 2010, 37, 334-338. [CrossRef]

37. Elsharkawy, H.A.; Li, L.; Esa, W.A.; Sessler, D.I.; Bashour, C.A. Outcome in patients who require venoarterial extracorporeal membrane oxygenation support after cardiac surgery. J. Cardiothorac. Vasc. Anesth. 2010, 24, 946-951. [CrossRef]

38. Hsu, P.S.; Chen, J.L.; Hong, G.J.; Tsai, Y.T.; Lin, C.Y.; Lee, C.Y.; Chen, Y.G.; Tsai, C.S. Extracorporeal membrane oxygenation for refractory cardiogenic shock after cardiac surgery: Predictors of early mortality and outcome from 51 adult patients. Eur. J. Cardiothorac. Surg. 2010, 37, 328-333. [CrossRef]

39. Lan, C.; Tsai, P.R.; Chen, Y.S.; Ko, W.J. Prognostic factors for adult patients receiving extracorporeal membrane oxygenation as mechanical circulatory support-A 14-year experience at a medical center. Artif. Organs 2010, 34, E59-E64. [CrossRef]

40. Rastan, A.J.; Dege, A.; Mohr, M.; Doll, N.; Falk, V.; Walther, T.; Mohr, F.W. Early and late outcomes of 517 consecutive adult patients treated with extracorporeal membrane oxygenation for refractory postcardiotomy cardiogenic shock. J. Thorac. Cardiovasc. Surg. 2010, 139, 302-311. [CrossRef]

41. Wu, V.C.; Tsai, H.B.; Yeh, Y.C.; Huang, T.M.; Lin, Y.F.; Chou, N.K.; Chen, Y.S.; Han, Y.Y.; Chou, A.; Lin, Y.H.; et al. Patients supported by extracorporeal membrane oxygenation and acute dialysis: Acute physiology and chronic health evaluation score in predicting hospital mortality. Artif. Organs 2010, 34, 828-835. [CrossRef] [PubMed]

42. Chen, Y.C.; Tsai, F.C.; Chang, C.H.; Lin, C.Y.; Jenq, C.C.; Juan, K.C.; Hsu, H.H.; Chang, M.Y.; Tian, Y.C.; Hung, C.C.; et al. Prognosis of patients on extracorporeal membrane oxygenation: The impact of acute kidney injury on mortality. Ann. Thorac. Surg. 2011, 91, 137-142. [CrossRef] [PubMed]

43. Bermudez, C.A.; Rocha, R.V.; Toyoda, Y.; Zaldonis, D.; Sappington, P.L.; Mulukutla, S.; Marroquin, O.C.; Toma, C.; Bhama, J.K.; Kormos, R.L. Extracorporeal membrane oxygenation for advanced refractory shock in acute and chronic cardiomyopathy. Ann. Thorac. Surg. 2011, 92, 2125-2131. [CrossRef] [PubMed]

44. Chang, W.W.; Tsai, F.C.; Tsai, T.Y.; Chang, C.H.; Jenq, C.C.; Chang, M.Y.; Tian, Y.C.; Hung, C.C.; Fang, J.T.; Yang, C.W.; et al. Predictors of mortality in patients successfully weaned from extracorporeal membrane oxygenation. PLoS ONE 2012, 7, e42687. [CrossRef] [PubMed]

45. Kim, T.H.; Lim, C.; Park, I.; Kim, D.J.; Jung, Y.; Park, K.H. Prognosis in the patients with prolonged extracorporeal membrane oxygenation. Korea J. Thorac. Cardiovasc. Surg. 2012, 45, 236-241. [CrossRef] [PubMed] 
46. Lee, S.H.; Chung, C.H.; Won Lee, J.; Ho Jung, S.; Choo, S.J. Factors predicting early- and long-term survival in patients undergoing extracorporeal membrane oxygenation (ECMO). J. Card. Surg. 2012, 27, $255-263$. [CrossRef] [PubMed]

47. Loforte, A.; Montalto, A.; Ranocchi, F.; Della Monica, P.L.; Casali, G.; Lappa, A.; Menichetti, A.; Contento, C.; Musumeci, F. Peripheral extracorporeal membrane oxygenation system as salvage treatment of patients with refractory cardiogenic shock: Preliminary outcome evaluation. Artif. Organs 2012, 36, E53-E61. [CrossRef] [PubMed]

48. Wu, M.Y.; Lee, M.Y.; Lin, C.C.; Chang, Y.S.; Tsai, F.C.; Lin, P.J. Resuscitation of non-postcardiotomy cardiogenic shock or cardiac arrest with extracorporeal life support: The role of bridging to intervention. Resuscitation 2012, 83, 976-981. [CrossRef]

49. Aubron, C.; Cheng, A.C.; Pilcher, D.; Leong, T.; Magrin, G.; Cooper, D.J.; Scheinkestel, C.; Pellegrino, V. Factors associated with outcomes of patients on extracorporeal membrane oxygenation support: A 5-year cohort study. Crit. Care 2013, 17, R73. [CrossRef]

50. Kielstein, J.T.; Heiden, A.M.; Beutel, G.; Gottlieb, J.; Wiesner, O.; Hafer, C.; Hadem, J.; Reising, A.; Haverich, A.; Kühn, C.; et al. Renal function and survival in 200 patients undergoing ECMO therapy. Nephrol. Dial. Transplant. 2013, 28, 86-90. [CrossRef]

51. Wu, M.Y.; Tseng, Y.H.; Chang, Y.S.; Tsai, F.C.; Lin, P.J. Using extracorporeal membrane oxygenation to rescue acute myocardial infarction with cardiopulmonary collapse: The impact of early coronary revascularization. Resuscitation 2013, 84, 940-945. [CrossRef] [PubMed]

52. Lazzeri, C.; Bernardo, P.; Sori, A.; Innocenti, L.; Passantino, S.; Chiostri, M.; Gensini, G.F.; Valente, S. Renal replacement therapy in patients with refractory cardiac arrest undergoing extracorporeal membrane oxygenation. Resuscitation 2013, 84, e121-e122. [CrossRef] [PubMed]

53. Unosawa, S.; Sezai, A.; Hata, M.; Nakata, K.; Yoshitake, I.; Wakui, S.; Kimura, H.; Takahashi, K.; Hata, H.; Shiono, M. Long-term outcomes of patients undergoing extracorporeal membrane oxygenation for refractory postcardiotomy cardiogenic shock. Surg. Today 2013, 43, 264-270. [CrossRef] [PubMed]

54. Xue, J.; Wang, L.; Chen, C.M.; Chen, J.Y.; Sun, Z.X. Acute kidney injury influences mortality in lung transplantation. Ren. Fail. 2014, 36, 541-545. [CrossRef] [PubMed]

55. Hsiao, C.C.; Chang, C.H.; Fan, P.C.; Ho, H.T.; Jenq, C.C.; Kao, K.C.; Chiu, L.C.; Lee, S.Y.; Hsu, H.H.; Tian, Y.C.; et al. Prognosis of patients with acute respiratory distress syndrome on extracorporeal membrane oxygenation: The impact of urine output on mortality. Ann. Thorac. Surg. 2014, 97, 1939-1944. [CrossRef] [PubMed]

56. Lee, S.W.; Yu, M.Y.; Lee, H.; Ahn, S.Y.; Kim, S.; Chin, H.J.; Na, K.Y. Risk Factors for Acute Kidney Injury and In-Hospital Mortality in Patients Receiving Extracorporeal Membrane Oxygenation. PLoS ONE 2015, 10, e0140674. [CrossRef] [PubMed]

57. Haneya, A.; Diez, C.; Philipp, A.; Bein, T.; Mueller, T.; Schmid, C.; Lubnow, M. Impact of Acute Kidney Injury on Outcome in Patients With Severe Acute Respiratory Failure Receiving Extracorporeal Membrane Oxygenation. Crit. Care Med. 2015, 43, 1898-1906. [CrossRef]

58. Huang, L.; Li, T.; Xu, L.; Hu, X.M.; Duan, D.W.; Li, Z.B.; Gao, X.J.; Li, J.; Wu, P.; Liu, Y.W. Extracorporeal Membrane Oxygenation Outcomes in Acute Respiratory Distress Treatment: Case Study in a Chinese Referral Center. Med. Sci. Monit. 2017, 23, 741-750. [CrossRef]

59. Antonucci, E.; Lamanna, I.; Fagnoul, D.; Vincent, J.L.; De Backer, D.; Silvio Taccone, F. The Impact of Renal Failure and Renal Replacement Therapy on Outcome During Extracorporeal Membrane Oxygenation Therapy. Artif. Organs 2016, 40, 746-754. [CrossRef]

60. Tsai, T.Y.; Chien, H.; Tsai, F.C.; Pan, H.C.; Yang, H.Y.; Lee, S.Y.; Hsu, H.H.; Fang, J.T.; Yang, C.W.; Chen, Y.C. Comparison of, R.I.F.L.E.; AKIN, and KDIGO classifications for assessing prognosis of patients on extracorporeal membrane oxygenation. J. Formos. Med. Assoc. 2017, 116, 844-851. [CrossRef]

61. Panholzer, B.; Meckelburg, K.; Huenges, K.; Hoffmann, G.; von der Brelie, M.; Haake, N.; Pilarczyk, K.; Cremer, J.; Haneya, A. Extracorporeal membrane oxygenation for acute respiratory distress syndrome in adults: An analysis of differences between survivors and non-survivors. Perfusion 2017, 32, 495-500. [CrossRef] [PubMed]

62. Chong, S.Z.; Fang, C.Y.; Fang, H.Y.; Chen, H.C.; Chen, C.J.; Yang, C.H.; Hang, C.L.; Yip, H.K.; Wu, C.J.; Lee, W.C. Associations with the In-Hospital Survival Following Extracorporeal Membrane Oxygenation in Adult Acute Fulminant Myocarditis. J. Clin. Med. 2018, 7, 452. [CrossRef] [PubMed] 
63. Devasagayaraj, R.; Cavarocchi, N.C.; Hirose, H. Does acute kidney injury affect survival in adults with acute respiratory distress syndrome requiring extracorporeal membrane oxygenation? Perfusion 2018, 33, 375-382. [CrossRef] [PubMed]

64. Liao, X.; Cheng, Z.; Wang, L.; Li, B. Analysis of the risk factors of acute kidney injury in patients receiving extracorporeal membrane oxygenation. Clin. Nephrol. 2018, 90, 270-275. [CrossRef] [PubMed]

65. Paek, J.H.; Park, S.; Lee, A.; Park, S.; Chin, H.J.; Na, K.Y.; Lee, H.; Park, J.T.; Kim, S. Timing for initiation of sequential continuous renal replacement therapy in patients on extracorporeal membrane oxygenation. Kidney Res. Clin. Pract. 2018, 37, 239-247. [CrossRef]

66. He, P.; Zhang, S.; Hu, B.; Wu, W. Retrospective study on the effects of the prognosis of patients treated with extracorporeal membrane oxygenation combined with continuous renal replacement therapy. Ann. Transl. Med. 2018, 6, 455. [CrossRef] [PubMed]

67. Zhang, H.; Wu, J.; Zou, D.; Xiao, X.; Yan, H.; Li, X.C.; Chen, W. Ablation of interferon regulatory factor 4 in T cells induces "memory" of transplant tolerance that is irreversible by immune checkpoint blockade. Am. J. Transplant. 2019, 19, 884-893. [CrossRef]

68. Kilburn, D.J.; Shekar, K.; Fraser, J.F. The Complex Relationship of Extracorporeal Membrane Oxygenation and Acute Kidney Injury: Causation or Association? BioMed Res. Int. 2016, 2016, 1094296. [CrossRef]

69. Cheng, R.; Hachamovitch, R.; Kittleson, M.; Patel, J.; Arabia, F.; Moriguchi, J.; Esmailian, F.; Azarbal, B. Complications of extracorporeal membrane oxygenation for treatment of cardiogenic shock and cardiac arrest: A meta-analysis of 1,866 adult patients. Ann. Thorac.Surg. 2014, 97, 610-616. [CrossRef]

70. Lin, C.Y.; Tsai, F.C.; Tian, Y.C.; Jenq, C.C.; Chen, Y.C.; Fang, J.T.; Yang, C.W. Evaluation of outcome scoring systems for patients on extracorporeal membrane oxygenation. Ann. Thorac. Surg. 2007, 84, 1256-1262. [CrossRef]

71. Moher, D.; Liberati, A.; Tetzlaff JAltman, D.G. Preferred reporting items for systematic reviews and meta-analyses: The PRISMA statement. PLoS Med. 2009, 6, e1000097. [CrossRef] [PubMed]

72. Bellomo, R.; Ronco, C.; Kellum, J.A.; Mehta, R.L. Palevsky PAcute renal failure-Definition outcome measures animal, m.o.d.e.l.s., fluid therapy and information technology needs: The Second International Consensus Conference of the Acute Dialysis Quality Initiative (ADQI) Group. Crit. Care 2004, 8, R204-R212. [CrossRef] [PubMed]

73. Mehta, R.L.; Kellum, J.A.; Shah, S.V.; Molitoris, B.A.; Ronco, C.; Warnock, D.G.; Levin, A. Acute Kidney Injury Network: Report of an initiative to improve outcomes in acute kidney injury. Crit. Care 2007, 11, R31. [CrossRef] [PubMed]

74. Section 2: AKI Definition. Kidney Int. 2012, 2, 19-36. [CrossRef] [PubMed]

75. DerSimonian, R.; Laird, N. Meta-analysis in clinical trials. Control. Clin. Trials 1986, 7, 177-188. [CrossRef]

76. Higgins, J.P.; Thompson, S.G.; Deeks, J.J.; Altman, D.G. Measuring inconsistency in meta-analyses. BMJ 2003, 327, 557-560. [CrossRef] [PubMed]

77. Easterbrook, P.J.; Gopalan, R.; Berlin, J.A.; Matthews, D.R. Publication bias in clinical research. Lancet 1991, 337, 867-872. [CrossRef]

78. McDonald, C.I.; Fraser, J.F.; Coombes, J.S.; Fung, Y.L. Oxidative stress during extracorporeal circulation. Eur. J. Cardiothorac. Surg. 2014, 46, 937-943. [CrossRef] [PubMed]

79. Ikeda, M.; Prachasilchai, W.; Burne-Taney, M.J.; Rabb, H.; Yokota-Ikeda, N. Ischemic acute tubular necrosis models and drug discovery: A focus on cellular inflammation. Drug Discov. Today 2006, 11, 364-370. [CrossRef] [PubMed]

80. Yimin, H.; Wenkui, Y.; Jialiang, S.; Qiyi, C.; Juanhong, S.; Zhiliang, L.; Changsheng, H.; Ning, L.; Jieshou, L. Effects of continuous renal replacement therapy on renal inflammatory cytokines during extracorporeal membrane oxygenation in a porcine model. J. Cardiothorac. Surg. 2013, 8, 113. [CrossRef] [PubMed]

81. McILwain, R.B.; Timpa, J.G.; Kurundkar, A.R.; Holt, D.W.; Kelly, D.R.; Hartman, Y.E.; Neel, M.L.; Karnatak, R.K.; Schelonka, R.L.; Anantharamaiah, G.M.; et al. Plasma concentrations of inflammatory cytokines rise rapidly during ECMO-related SIRS due to the release of preformed stores in the intestine. Lab. Investig. 2010, 90, 128-139. [CrossRef] [PubMed]

82. Reed, R.C.; Rutledge, J.C. Laboratory and clinical predictors of thrombosis and hemorrhage in 29 pediatric extracorporeal membrane oxygenation nonsurvivors. Pediatr. Dev. Pathol. 2010, 13, 385-392. [CrossRef] [PubMed] 
83. Williams, D.C.; Turi, J.L.; Hornik, C.P.; Bonnadonna, D.K.; Williford, W.L.; Walczak, R.J.; Watt, K.M.; Cheifetz, I.M. Circuit oxygenator contributes to extracorporeal membrane oxygenation-induced hemolysis. ASAIO J. 2015, 61, 190-195. [CrossRef] [PubMed]

84. Askenazi, D.J.; Selewski, D.T.; Paden, M.L.; Cooper, D.S.; Bridges, B.C.; Zappitelli, M.; Fleming, G.M. Renal replacement therapy in critically ill patients receiving extracorporeal membrane oxygenation. Clin. J. Am. Soc. Nephrol. 2012, 7, 1328-1336. [CrossRef] [PubMed]

85. Lubnow, M.; Philipp, A.; Foltan, M.; Enger, T.B.; Lunz, D.; Bein, T.; Haneya, A.; Schmid, C.; Riegger, G.; Müller, T.; et al. Technical complications during veno-venous extracorporeal membrane oxygenation and their relevance predicting a system-exchange-Retrospective analysis of 265 cases. PLOS ONE 2014, 9, e112316. [CrossRef] [PubMed]

86. Murphy, D.A.; Hockings, L.E.; Andrews, R.K.; Aubron, C.; Gardiner, E.E.; Pellegrino, V.A.; Davis, A.K. Extracorporeal membrane oxygenation-hemostatic complications. Transfus. Med. Rev. 2015, 29, 90-101. [CrossRef]

87. Lehle, K.; Philipp, A.; Zeman, F.; Lunz, D.; Lubnow, M.; Wendel, H.P.; Göbölös, L.; Schmid, C.; Müller, T. Technical-Induced Hemolysis in Patients with Respiratory Failure Supported with Veno-Venous ECMO_Prevalence and Risk Factors. PLoS ONE 2015, 10, e0143527. [CrossRef] [PubMed]

88. Adademir, T.; Ak, K.; Aljodi, M.; Elçi, M.E.; Arsan, S.; Isbir, S. The effects of pulsatile cardiopulmonary bypass on acute kidney injury. Int. J. Artif. Organs 2012, 35, 511-519. [CrossRef]

89. Abu-Omar, Y.; Ratnatunga, C. Cardiopulmonary bypass and renal injury. Perfusion 2006, 21, $209-213$. [CrossRef]

90. Santana-Santos, E.; Marcusso, M.E.; Rodrigues, A.O.; Queiroz, F.G.; Oliveira, L.B.; Rodrigues, A.R.; Palomo, J.D. Strategies for prevention of acute kidney injury in cardiac surgery: An integrative review. Revista Brasileira Terpia Intensiva 2014, 26, 183-192. [CrossRef]

91. Thongprayoon, C.; Kaewput, W.; Thamcharoen, N.; Bathini, T.; Watthanasuntorn, K.; Lertjitbanjong, P.; Sharma, K.; Salim, S.A.; Ungprasert, P.; Wijarnpreecha, K.; et al. Incidence and Impact of Acute Kidney Injury after Liver Transplantation: A Meta-Analysis. J. Clin. Med. 2019, 8, 372. [CrossRef] [PubMed]

92. Thongprayoon, C.; Kaewput, W.; Thamcharoen, N.; Bathini, T.; Watthanasuntorn, K.; Salim, S.A.; Ungprasert, P.; Lertjitbanjong, P.; Aeddula, N.R.; Torres-Ortiz, A.; et al. Acute Kidney Injury in Patients Undergoing Total Hip Arthroplasty: A Systematic Review and Meta-Analysis. J. Clin. Med. 2019, 8, 66. [CrossRef] [PubMed]

93. Thongprayoon, C.; Cheungpasitporn, W.; Mao, M.A.; Harrison, A.M.; Erickson, S.B. Elevated admission serum calcium phosphate product as an independent risk factor for acute kidney injury in hospitalized patients. Hosp. Pract. (1995) 2019, 47, 73-79. [CrossRef] [PubMed]

94. Thongprayoon, C.; Cheungpasitporn, W.; Mao, M.A.; Sakhuja, A.; Kashani, K. U-shape association of serum albumin level and acute kidney injury risk in hospitalized patients. PLoS ONE 2018, 13, e0199153. [CrossRef] [PubMed]

95. Thongprayoon, C.; Cheungpasitporn, W.; Mao, M.A.; Sakhuja, A.; Erickson, S.B. Admission calcium levels and risk of acute kidney injury in hospitalised patients. Int. J. Clin. Pract. 2018, 72, e13057. [CrossRef] [PubMed]

96. Thongprayoon, C.; Cheungpasitporn, W.; Mao, M.A.; Sakhuja, A.; Erickson, S.B. Admission hyperphosphatemia increases the risk of acute kidney injury in hospitalized patients. J. Nephrol. 2018, 31, 241-247. [CrossRef] [PubMed]

97. Razo-Vazquez, A.O.; Thornton, K. Extracorporeal Membrane Oxygenation-What the Nephrologist Needs to Know. Adv. Chronic Kidney Dis. 2016, 23, 146-151. [CrossRef]

98. Pedersen, T.H.; Videm, V.; Svennevig, J.L.; Karlsen, H.; Østbakk, R.W.; Jensen, Ø.; Mollnes, T.E. Extracorporeal membrane oxygenation using a centrifugal pump and a servo regulator to prevent negative inlet pressure. Ann. Thorac. Surg. 1997, 63, 1333-1339. [CrossRef]

99. Kress, D.C.; Cohen, D.J.; Swanson, D.K.; Hegge, J.O.; Young, J.W.; Watson, K.M.; Rasmussen, P.W.; Berkoff, H.A. Pump-induced hemolysis in a rabbit model of neonatal ECMO. ASAIO Trans. 1987, 33, 446-452.

100. Lou, S.; MacLaren, G.; Best, D.; Delzoppo, C.; Butt, W. Hemolysis in pediatric patients receiving centrifugal-pump extracorporeal membrane oxygenation: Prevalence, risk factors, and outcomes. Crit. Care Med. 2014, 42, 1213-1220. [CrossRef] 
101. Toomasian, J.M.; Bartlett, R.H. Hemolysis and ECMO pumps in the 21st Century. Perfusion 2011, 26, 5-6. [CrossRef] [PubMed]

102. Thongprayoon, C.; Cheungpasitporn, W.; Kittanamongkolchai, W.; Harrison, A.M.; Kashani, K. Prognostic Importance of Low Admission Serum Creatinine Concentration for Mortality in Hospitalized Patients. Am. J. Med. 2017, 130, 545-554. [CrossRef] [PubMed]

103. Thongprayoon, C.; Cheungpasitporn, W.; Kashani, K. Serum creatinine level, a surrogate of muscle mass, predicts mortality in critically ill patients. J. Thorac. Dis. 2016, 8, E305-E311. [CrossRef] [PubMed]

104. Thongprayoon, C.; Cheungpasitporn, W.; Kittanamongkolchai, W.; Srivali, N.; Ungprasert, P.; Kashani, K. Optimum methodology for estimating baseline serum creatinine for the acute kidney injury classification. Nephrology (Carlton) 2015, 20, 881-886. [CrossRef] [PubMed]

105. Vaidya, V.S.; Ramirez, V.; Ichimura, T.; Bobadilla, N.A.; Bonventre, J.V. Urinary kidney injury molecule-1: A sensitive quantitative biomarker for early detection of kidney tubular injury. Am. J. Physiol. Renal Physiol. 2006, 290, F517-F529. [CrossRef]

106. Mishra, J.; Ma, Q.; Prada, A.; Mitsnefes, M.; Zahedi, K.; Yang, J.; Barasch, J.; Devarajan, P. Identification of neutrophil gelatinase-associated lipocalin as a novel early urinary biomarker for ischemic renal injury. J. Am. Soc. Nephrol. 2003, 14, 2534-2543. [CrossRef]

107. Hosohata, K.; Ando, H.; Fujimura, A. Urinary vanin-1 as a novel biomarker for early detection of drug-induced acute kidney injury. J. Pharmacol. Exp. Ther. 2012, 341, 656-662. [CrossRef]

108. Kashani, K.; Cheungpasitporn, W.; Ronco, C. Biomarkers of acute kidney injury: The pathway from discovery to clinical adoption. Clin. Chem. Lab. Med. 2017, 55, 1074-1089. [CrossRef]

(C) 2019 by the authors. Licensee MDPI, Basel, Switzerland. This article is an open access article distributed under the terms and conditions of the Creative Commons Attribution (CC BY) license (http://creativecommons.org/licenses/by/4.0/). 